\title{
RELATIVE HOMOLOGICAL ALGEBRA
}

\author{
BY \\ G. HOCHSCHILD
}

Introduction. The main purpose of this paper is to draw attention to certain functors, exactly analogous to the functors "Tor" and "Ext" of Cartan-Eilenberg [2], but applicable to a module theory that is relativized with respect to a given subring of the basic ring of operators. In particular, we shall show how certain relative cohomology theories for groups, rings, and Lie algebras can be subsumed under the theory of the relative Ext functor, just as (in [2]) the ordinary cohomology theories have been subsumed under the theory of the ordinary Ext functor.

Among the various relative cohomology groups that have been considered so far, some can be expressed in terms of the ordinary Ext functor; these have been studied systematically within the framework of general homological algebra by $M$. Auslander (to appear). A typical feature of these relative groups is that they appear naturally as terms of exact sequences whose other terms are the ordinary cohomology groups of the algebraic system in question, and of its given subsystem.

There is, however, another type of relative cohomology theory whose groups are not so intimately linked to the ordinary cohomology groups and exhibit a more individualized behaviour. Specifically, the relative cohomology groups for Lie algebras, as defined (in [3]) by Chevalley and Eilenberg, and the relative cohomology groups of groups, defined and investigated by $\mathrm{I}$. T. Adamson [1], are of this second type. It is these more genuinely "relative" cohomology theories that fall in our present framework of relative homological algebra.

Our plan here is to sketch the general features of the relative Tor and Ext functors ( $\$ 2)$ and to illustrate some of their possible uses or interpretations by a selection of unelaborated examples. Thus, $\$ 3$ illustrates the use of the relative Ext functor in extending the cohomology theory for algebras. $\$ 4$ deals with relative homology and relative cohomology of groups, and involves both the relative Tor functor and the relative Ext functor. $\$ 5$ discusses the role played by the relative Ext functor in the cohomology theory for Lie algebras.

Since this paper is intended to serve as a preliminary survey, and since the topics dealt with are supplementary to the corresponding topics of the nonrelative theory (contained in [2]), we feel justified in presupposing that the

Received by the editors January 20, 1956. 
reader is familiar with the elementary technique and the general notions of homological algebra.

1. Relatively projective and injective modules. Let $R$ be a ring with an identity element, 1 , and let $S$ be a subring of $R$ containing 1 . All the $R$-modules we shall consider are assumed to be "unitary," in the sense that 1 acts as the identity operator. An $R$-module will be regarded also as an $S$-module, in the natural way.

An exact sequence of $R$-homomorphisms between $R$-modules, $t_{i}: M_{i} \rightarrow M_{i-1}$ (or $t^{i}: M^{i} \rightarrow M^{i+1}$, with appropriate changes below) is called $(R, S)$-exact if, for each $i$, the kernel of $t_{i}$ is a direct $S$-module summand of $M_{i}$. Clearly, a sequence of $R$-homomorphisms $t_{i}$ is $(R, S)$-exact if and only if, for each $i$, (1): $t_{i} \circ t_{i+1}=0$, and (2): there exists an $S$-homotopy, i.e., a sequence of $S$-homomorphisms $h_{i}: M_{i} \rightarrow M_{i+1}$ such that $t_{i+1} \circ h_{i}+h_{i-1} \circ t_{i}$ is the identity map of $M_{i}$ onto itself.

An $R$-module $A$ is said to be $(R, S)$-injective if, for every $(R, S)$-exact sequence (0) $\rightarrow U \rightarrow_{p} V \rightarrow_{q} W \rightarrow(0)$, and every $R$-homomorphism $h$ of $U$ into $A$, there is an $R$-homomorphism $h^{\prime}$ of $V$ into $A$ such that $h^{\prime} \circ p=h$. Dually, $A$ is said to be $(R, S)$-projective if, for every such sequence, and every $R$-homomorphism $g$ of $A$ into $W$, there is an $R$-homomorphism $g^{\prime}$ of $A$ into $V$ such that $q \circ g^{\prime}=g\left({ }^{1}\right)$.

Let $t_{i}: M_{i} \rightarrow M_{i-1}$ be any $(R, S)$-exact sequence. It follows from our definitions and from the exactness properties of the functor $\mathrm{Hom}_{R}$ that the induced sequence of homomorphisms $\operatorname{Hom}_{R}\left(M_{i-1}, A\right) \rightarrow \operatorname{Hom}_{R}\left(M_{i}, A\right), h \rightarrow h \circ t_{i}$, is exact whenever $A$ is $(R, S)$-injective. Similarly, the induced sequence of homomorphisms $\operatorname{Hom}_{R}\left(A, M_{i}\right) \rightarrow \operatorname{Hom}_{R}\left(A, M_{i-1}\right), h \rightarrow t_{i} \circ h$, is exact whenever $A$ is $(R, S)$-projective.

Let $A$ be an $S$-module, and consider the group $\operatorname{Hom}_{S}(R, A)$ of all $S$-homomorphisms of $R$ into $A$, i.e., of all maps $f: R \rightarrow A$ such that $f(s r)=s \cdot f(r)$, for all $s \in S$ and all $r \in R$. This group is made into an $R$-module by defining, for $r$ and $r^{\prime}$ in $R,(r \cdot f)\left(r^{\prime}\right)=f\left(r^{\prime} r\right)$.

Lemma 1. For every $S$-module $A$, the $R$-module $\operatorname{Hom}_{S}(R, A)$ is $(R, S)$ injective.

Proof. Let $(0) \rightarrow U \rightarrow_{p} V \rightarrow_{q} W \rightarrow(0)$ be an $(R, S)$-exact sequence. Since $p$ maps $U$ isomorphically onto a direct $S$-module summand of $V$, it is clear that the map $h \rightarrow h \circ p$ sends $\operatorname{Hom}_{S}(V, A)$ onto $\operatorname{Hom}_{s}(U, A)$. On the other hand, for any $R$-module $M$, there is a natural isomorphism of the group $\operatorname{Hom}_{R}\left(M, \operatorname{Hom}_{S}(R, A)\right)$ onto $\operatorname{Hom}_{S}(M, A) ; k \rightarrow k_{1}$, where $k_{1}(m)=k(m)(1)$, for

(1) If $\phi$ is a homomorphism of a ring $T$ into $R$, and $S=\phi(T)$, then the $(R, S)$-injective (projective) modules are precisely the $\phi$-injective (projective) modules, in the sense of CartanEilenberg, [2, Chap. II, \$6]. In fact, the equivalence of these notions follows from Lemmas 1 and 2 below, and these lemmas themselves amount to the same as Proposition 6.3, Chap. II, of $[2]$. 
every $m \in M$. Using this, with $M=V$, and with $M=U$, we conclude that the map $k \rightarrow k \circ p$ sends $\operatorname{Hom}_{R}\left(V, \operatorname{Hom}_{S}(R, A)\right)$ onto $\operatorname{Hom}_{R}\left(U, \operatorname{Hom}_{S}(R, A)\right)$, which means precisely that $\operatorname{Hom}_{S}(R, A)$ is $(R, S)$-injective.

The natural standard identification of an $R$-module $M$ with the $R$-module $\operatorname{Hom}_{R}(R, M) \subset \operatorname{Hom}_{S}(R, M)$, under which each element $m \in M$ corresponds to the homomorphism $m^{\prime}$, where $m^{\prime}(r)=r \cdot m$, for every $r \in R$, gives rise to the natural exact sequence

$$
(0) \rightarrow M \rightarrow \operatorname{Hom}_{S}(R, M) \rightarrow \operatorname{Hom}_{S}(R, M) / \operatorname{Hom}_{R}(R, M) \rightarrow(0) .
$$

On the other hand, the map $h \rightarrow h(1)$ is an $S$-homomorphism $\operatorname{Hom}_{S}(R, M) \rightarrow M$, sending $m^{\prime}$ back onto $m$. It follows that our exact sequence is actually $(R, S)$-exact. If $M$ is $(R, S)$-injective, it follows at once that our sequence is $(R, R)$-exact, i.e., that $\operatorname{Hom}_{R}(R, M)$ is an exact $R$-module summand of $\operatorname{Hom}_{S}(R, M)$. Since a direct $R$-module summand of an $(R, S)$-injective module is still $(R, S)$-injective, we conclude that an $R$-module $M$ is $(R, S)$-injective if and only if $\operatorname{Hom}_{R}(R, M)$ is a direct $R$-module summand of $\operatorname{Hom}_{S}(R, M)$.

Proposition 1. Let $M$ be an $(R, S)$-injective $R$-module, and suppose that $U \rightarrow V$ is a homomorphism of $R$-modules such that the induced map $\operatorname{Hom}_{S}(V, M)$ $\rightarrow \operatorname{Hom}_{S}(U, M)$ is an epimorphism. Then the map $\operatorname{Hom}_{R}(V, M) \rightarrow \operatorname{Hom}_{R}(U, M)$ is also an epimorphism.

Proof. We have seen above that there is an $R$-projection of $\operatorname{Hom}_{S}(R, M)$ onto $\operatorname{Hom}_{R}(R, M)$. We identify $\operatorname{Hom}_{R}(R, M)$ with $M$ and note that such an $R$-projection induces an epimorphism $\operatorname{Hom}_{R}\left(B, \operatorname{Hom}_{S}(R, M)\right) \rightarrow \operatorname{Hom}_{R}(B, M)$, for every $R$-module $B$. If we compose this with the natural isomorphism of $\operatorname{Hom}_{S}(B, M)$ onto $\operatorname{Hom}_{R}\left(B, \operatorname{Hom}_{S}(R, M)\right.$ ) (whose inverse we defined in the proof of Lemma 1) we obtain an epimorphism $\operatorname{Hom}_{S}(B, M) \rightarrow \operatorname{Hom}_{R}(B, M)$. Moreover, this epimorphism is natural with respect to $B$, in the sense of the theory of functors. Hence, using this with $B=V$ and with $B=U$, we obtain an exact and commutative diagram

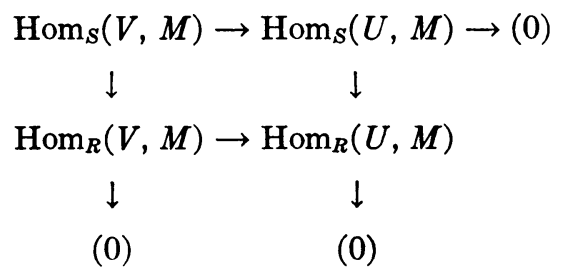

from which it follows at once that the map $\operatorname{Hom}_{R}(V, M) \rightarrow \operatorname{Hom}_{R}(U, M)$ is an epimorphism.

Lemma 2. For every $S$-module $A$, the $R$-module $R \otimes_{S} A$ is $(R, S)$-projective.

Proof. Let $(0) \rightarrow U \rightarrow_{p} V \rightarrow_{q} W \rightarrow(0)$ be an $(R, S)$-exact sequence. Since the kernel of $q$ is a direct $S$-module summand of $V$, the map $h \rightarrow q \circ h$ sends 
$\operatorname{Hom}_{S}(A, V)$ onto $\operatorname{Hom}_{S}(A, W)$. On the other hand, for any $R$-module $M$, there is a natural isomorphism of $\operatorname{Hom}_{R}\left(R \otimes_{S} A, M\right)$ onto $\operatorname{Hom}_{S}(A, M)$; $k \rightarrow k_{1}$, where $k_{1}(a)=k(1 \otimes a)$, for every $a \in A$. Using this, with $M=V$, and with $M=W$, we conclude that the map $k \rightarrow q \circ k$ sends $\operatorname{Hom}_{R}\left(R \otimes{ }_{S} A, V\right)$ onto $\operatorname{Hom}_{R}\left(R \otimes_{S} A, W\right)$, which means precisely that $R \otimes_{S} A$ is $(R, S)$-projective.

If $M$ is any $R$-module, the natural map $R \otimes{ }_{S} M \rightarrow M$ gives rise to an exact sequence $(0) \rightarrow K_{M} \rightarrow R \otimes{ }_{S} M \rightarrow M \rightarrow(0)$. The map $m \rightarrow 1 \otimes m$ is an $S$-isomorphism of $M$ onto an $S$-module complement of $K_{M}$ in $R \otimes_{S} M$, showing that our exact sequence is actually $(R, S)$-exact. If $M$ is $(R, S)$-projective it follows that the sequence is $(R, R)$-exact, so that $M$ is $R$-isomorphic with a direct $R$-module summand of $R \otimes_{S} M$. Since a direct $R$-module summand of an $(R, S)$-projective module is still $(R, S)$-projective, we may now conclude that an $R$-module $M$ is $(R, S)$-projective if and only if it is $R$-isomorphic with a direct $R$-module summand of $R \otimes_{S} M$, or if and only if $K_{M}$ is a direct $R$ module summand of $R \otimes_{S} M$.

Proposition 2. Let $M$ be an $(R, S)$-projective $R$-module; and suppose that $V \rightarrow W$ is a homomorphism of $R$-modules such that the induced map $\operatorname{Hom}_{S}(M, V)$ $\rightarrow \operatorname{Hom}_{S}(M, W)$ is an epimorphism. Then the induced map $\operatorname{Hom}_{R}(M, V)$ $\rightarrow \operatorname{Hom}_{R}(M, W)$ is also an epimorphism.

Proof. We have seen above that there is an $R$-isomorphism of $M$ onto a direct $R$-module summand of $R \otimes_{S} M$. For every $R$-module $B$, this induces an epimorphism $\operatorname{Hom}_{R}\left(R \otimes_{S} M, B\right) \rightarrow \operatorname{Hom}_{R}(M, B)$. If we compose this with the natural isomorphism of $\operatorname{Hom}_{S}(M, B)$ onto $\operatorname{Hom}_{R}\left(R \otimes_{S} M, B\right)$ (whose inverse we defined in the proof of Lemma 2)' we obtain an epimorphism $\operatorname{Hom}_{S}(M, B) \rightarrow \operatorname{Hom}_{R}(M, B)$. Moreover, this epimorphism is natural with respect to $B$. Hence, using this with $B=V$ and with $B=W$, we obtain an exact and commutative diagram

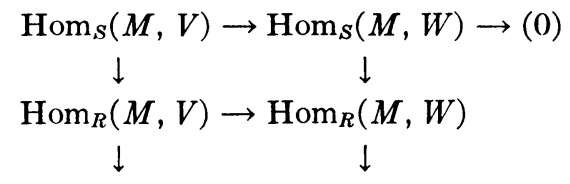

$(0)$

(0)

from which we see that the map $\operatorname{Hom}_{R}(M, V) \rightarrow \operatorname{Hom}_{R}(M, W)$ is an epimorphism.

Proposition 3. Let $M$ be an $(R, S)$-projective $R$-module, and suppose that $U \rightarrow V$ is a homomorphism of right $R$-modules such that the induced map $U \otimes_{S} M \rightarrow V \otimes_{S} M$ is a monomorphism. Then the map $U \otimes_{R} M \rightarrow V \otimes_{R} M$ is also a monomorphism.

Proof. There is an $R$-isomorphism of $M$ onto a direct $R$-module summand 
of $R \otimes_{S} M$. For every right $R$-module $B$, this induces a monomorphism $B \otimes_{R} M \rightarrow B \otimes_{R}\left(R \otimes_{S} M\right)=B \otimes_{S} M$. Using this, with $B=U$ and with $B=V$, we obtain an exact and commutative diagram

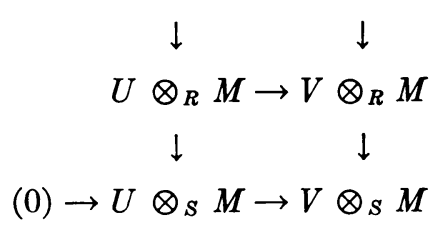

from which we see at once that the map $U \otimes_{R} M \rightarrow V \otimes_{R} M$ is a monomorphism.

2. The relative Tor and Ext functors. By an $(R, S)$-projective resolution of an $R$-module $M$ we shall mean an $(R, S)$-exact sequence $\rightarrow C_{1} \rightarrow C_{0} \rightarrow M \rightarrow(0)$ in which each $C_{i}$ is $(R, S)$-projective. It follows at once from Lemma 2 and the remarks following its proof that every $R$-module has an $(R, S)$ projective resolution. In fact, we may take $C_{0}=R \otimes_{S} M$, with the natural map $R \otimes_{S} M \rightarrow M$, and then proceed in the same way from the kernel of this map in order to obtain $C_{1} \rightarrow C_{0}$, etc. The $(R, S)$-projective resolution obtained from this process is called the $\operatorname{standard}(R, S)$-projective resolution of $M$.

Let $B$ be an ordinary (left) $R$-module, and let $A$ be a right $R$-module. Let $\cdots \rightarrow X_{1} \rightarrow X_{0} \rightarrow A \rightarrow(0)$ be the standard (right) $(R, S)$-projective resolution of $A$. Let $u_{i}$ denote the $R$-homomorphism $X_{i} \rightarrow X_{i-1}$ of this resolution, for $i>0$; but let $u_{0}$ stand for the 0 -map on $X_{0}$. It is convenient to introduce the graded $R$-module $X$ whose homogeneous components of non-negative degrees are the $X_{i}$, while its homogeneous components of negative degrees are (0). Also, denote by $u$ the homogeneous $R$-endomorphism of degree -1 on $X$ whose restriction to $X_{i}$ is $u_{i}$. Thus we have a graded right $R$-complex $(X, u)$. Likewise, from the standard $(R, S)$-projective resolution $\rightarrow Y_{1} \rightarrow Y_{0} \rightarrow B \rightarrow(0)$ of $B$, we obtain the graded (left) $R$-complex $(Y, v)$.

Now form the graded group $Z=X \otimes_{R} Y$, with homogeneous components $Z_{n}=\sum_{i+j=n} X_{i} \otimes_{R} Y_{j}$, and define the homogeneous endomorphism $w$ of degree -1 on $Z$ such that, for $x \in X_{i}$ and $y \in Y$,

$$
w(x \otimes y)=u(x) \otimes y+(-1)^{i} x \otimes v(y) .
$$

Since $u^{2}=0$ and $v^{2}=0$, it follows that $w^{2}=0$, and we have a graded group complex $(Z, w)$. We define $\operatorname{Tor}_{n}^{(R, S)}(A, B)$ as the $n$th homology group $H_{n}(Z, w)$ of this complex. Clearly, these groups are $(0)$ for $n<0$.

Suppose we have two $R$-modules $M$ and $N$, and two sequences of $R$ homomorphisms $\cdots \rightarrow C_{1} \rightarrow C_{0} \rightarrow M \rightarrow(0)$, and $\cdots \rightarrow D_{1} \rightarrow D_{0} \rightarrow N \rightarrow(0)$, satisfying the following conditions: (1): in each sequence, the composite of successive maps is 0 ; (2): the $C$-sequence has an $S$-homotopy; (3): each $D_{i}$ is $(R, S)$-projective. Suppose also that we are given an $R$-homomorphism 
$q: N \rightarrow M$. Then it is easily seen that we can successively find $R$-homomorphisms $D_{i} \rightarrow C_{i}$ such that the resulting diagram

$$
\begin{aligned}
& \cdots \rightarrow C_{1} \rightarrow C_{0} \rightarrow M \rightarrow(0) \\
& \uparrow \uparrow \uparrow q \\
& \cdots \rightarrow D_{1} \rightarrow D_{0} \rightarrow N \rightarrow(0)
\end{aligned}
$$

is commutative. Moreover, if $\left(s_{i}\right)$ and $\left(t_{i}\right)$ are any two such systems of $R$ homomorphisms, there is an $R$-homotopy connecting them, i.e., a sequence of $R$-homomorphisms $h_{i}: D_{i} \rightarrow C_{i+1}$ such that, if $c_{i}$ and $d_{i}$ are the maps of the given sequences, $s_{i}-t_{i}=c_{i+1} \circ h_{i}+h_{i-1} \circ d_{i}$, for all $i$ (where $h_{-1}=0$ ).

This is enough information to enable one to proceed exactly as in the usual theory of $\operatorname{Tor}^{R}$ and thus establish the following facts. Every pair of $R$-homomorphisms $A \rightarrow A^{\prime}$ and $B \rightarrow B^{\prime}$ induces a unique homomorphism $\operatorname{Tor}_{n}^{(R, S)}(A, B) \rightarrow \operatorname{Tor}_{n}^{(R, S)}\left(A^{\prime}, B^{\prime}\right)$, and these induced homomorphisms have all the requisite properties to make $\operatorname{Tor}_{n}^{(R, S)}$ a twice covariant functor. Moreover, the actual choice of the $(R, S)$-projective resolutions of $A$ and $B$ that are used in computing $\operatorname{Tor}_{n}^{(R, S)}(A, B)$ has, to within natural isomorphisms, no influence on the result. In particular, one sees from this that, for $n>0$, $\operatorname{Tor}_{n}^{(R, S)}(A, B)=(0)$, whenever either $A$ or $B$ is $(R, S)$-projective. On the other hand, it can be seen directly from the definition that, for arbitrary $A$ and $B$, $\operatorname{Tor}_{0}^{(R, S)}(A, B)=A \otimes_{R} B$.

As in the case of $\operatorname{Tor}^{R}$, it is actually sufficient to make a resolution of only one of the modules; $\operatorname{Tor}_{n}^{(R, S)}(A, B)$ may be identified with the homology group $H_{n}\left(A \otimes_{R} Y\right)$ of the complex $A \otimes_{R} Y$ that is defined as above, using, in the place of $X$, the complex whose component of degree 0 is $A$ and all whose other components are (0). Similarly, $\operatorname{Tor}_{n}^{(R, S)}(A, B)=H_{n}\left(X \otimes_{R} B\right)$. This can be proved by the methods of Chapter $\mathrm{V}$ of [2]. The critical fact that is needed is the following immediate consequence of Proposition 3 above: if $A$ is $(R, S)$-projective then, for any $(R, S)$-exact sequence $\cdots \rightarrow B_{i} \rightarrow B_{i-1} \rightarrow \cdots$, the induced sequence $\cdots \rightarrow A \otimes_{R} B_{i} \rightarrow A \otimes_{R} B_{i-1} \rightarrow \cdots$ is exact (similarly, with the roles of the left and right modules interchanged). We shall briefly sketch a direct proof of the above.

Consider the subcomplex $X^{\prime}$ of $X$ whose components are given by: $X_{i}^{\prime}=X_{i}$, for $i>0 ; X_{0}^{\prime}=u_{1}\left(X_{1}\right)$. The injection $X^{\prime} \rightarrow X$ and the $R$-homomorphism $X_{0} \rightarrow A$ of our resolution compose to the $(R, S)$-exact sequence $(0) \rightarrow X^{\prime}$ $\rightarrow X \rightarrow A \rightarrow(0)$. Since $Y$ is $(R, S)$-projective, the induced sequence $(0) \rightarrow X^{\prime} \otimes_{R} Y$ $\rightarrow X \otimes_{R} Y \rightarrow A \otimes_{R} Y \rightarrow(0)$ is exact. Clearly, the homomorphisms of this sequence are compatible with the structure of a complex of each term. Hence if we show that the homology groups of the complex $X^{\prime} \otimes_{R} Y$ are (0) it will follow that the map $X \otimes_{R} Y \rightarrow A \otimes_{R} Y$ induces an isomorphism of $H_{n}\left(X \otimes_{R} Y\right)$ onto $H_{n}\left(A \otimes_{R} Y\right)$, establishing the above claim concerning $\operatorname{Tor}_{n}^{(R, S)}(A, B)$. In order to show that $H\left(X^{\prime} \otimes_{R} Y\right)=(0)$ we define an increasing sequence of sub- 
complexes $Z^{k}$ of $X^{\prime} \otimes_{R} Y$ whose components are given by:

$$
Z_{n}^{k}=\sum_{j \leqq k} X_{n-j}^{\prime} \otimes_{R} Y_{j}
$$

Then each factor complex $Z^{k} / Z^{k-1}$ is isomorphic with $X^{\prime} \otimes_{R} Y_{k}$. Since $X^{\prime}$ has an $S$-homotopy and $Y_{k}$ is $(R, S)$-projective, it follows that the homology groups of $Z^{k} / Z^{k-1}$ are (0). Hence the injections $Z^{k-1} \rightarrow Z^{k}$ induce isomorphisms of $H\left(Z^{k-1}\right)$ onto $H\left(Z^{k}\right)$. Since $Z^{k}=(0)$, for $k<0$, we conclude that each $H\left(Z^{k}\right)=(0)$. Since $H_{n}\left(Z^{k}\right)=H_{n}\left(X^{\prime} \otimes_{R} Y\right)$, as soon as $k>n$, we conclude that $H\left(X^{\prime} \otimes_{R} Y\right)=(0)$.

Let $(0) \rightarrow U \rightarrow V \rightarrow W \rightarrow(0)$ be an $(R, S)$-exact sequence. This leads (as above) to an exact sequence of complexes $(0) \rightarrow U \otimes_{R} Y \rightarrow V \otimes_{R} Y \rightarrow W \otimes_{R} Y$ $\rightarrow(0)$. Using that $\operatorname{Tor}_{n}^{(R, S)}(A, B)=H_{n}\left(A \otimes_{R} Y\right)$, we obtain the exact sequence $\rightarrow \operatorname{Tor}_{n}^{(R, S)}(U, B) \rightarrow \operatorname{Tor}_{n}^{(R, S)}(V, B) \rightarrow \operatorname{Tor}_{n}^{(R, S)}(W, B) \rightarrow \operatorname{Tor}_{n-1}^{(R, S)}(U, B) \rightarrow$ and, clearly, there is a similar exact sequence with the roles of left and right modules interchanged.

Now we pass to the discussion of the relative Ext functor. By an $(R, S)$ injective resolution of an $R$-module $M$ we shall mean an $(R, S)$-exact sequence $(0) \rightarrow M \rightarrow C^{0} \rightarrow C^{1} \rightarrow \cdots$ in which each $C^{i}$ is $(R, S)$-injective. It follows from Lemma 1 and the remarks following its proof that every $R$-module has an $(R, S)$-injective resolution. In fact, we may take $C^{0}=\operatorname{Hom}_{S}(R, M)$, with the monomorphism $M \rightarrow \operatorname{Hom}_{S}(R, M)$ that we discussed in connection with Lemma 1, and proceed in the same way with the cokernel $C^{0} / \operatorname{Hom}_{R}(R, M)$ of this monomorphism, etc. The $(R, S)$-injective resolution so obtained will be called the standard $(R, S)$-injective resolution of $M$.

Let $A$ and $B$ be $R$-modules, and let $(X, u)$ be the $R$-complex obtained (as above) from the standard $(R, S)$-projective resolution of $A$. If $(0) \rightarrow B$ $\rightarrow Y^{0} \rightarrow_{v^{0}} Y^{1} \rightarrow_{v^{1}} \ldots$ is the standard $(R, S)$-injective resolution of $B$ we denote by $(Y, v)$ the $R$-complex formed by the $Y^{i}$ and the maps $v^{i}$, agreeing that $Y^{i}=(0)$, for $i<0$. Now form the graded group $Z$ whose homogeneous components are the direct sums $Z^{n}=\sum_{i+j=n} \operatorname{Hom}_{R}\left(X_{i}, Y^{j}\right)$. Define a homogeneous endomorphism $w$ of degree 1 on $Z$ such that, for $h \in \operatorname{Hom}_{R}\left(X_{i}, Y^{j}\right)$, $w(h)=h \circ u_{i+1}+(-1)^{i} v^{i} \circ h$. Then $w^{2}=0$, and $(Z, w)$ is a graded group complex. We define $\operatorname{Ext}_{(R, S)}^{n}(A, B)$ as the $n$th cohomology group $H^{n}(Z, w)$ of this complex. Clearly, these groups are $(0)$, for $n<0$.

Using the result on the existence and uniqueness (up to homotopies) of maps between complexes that we have cited in our above discussion of $\operatorname{Tor}_{n}^{(R, S)}$, as well as the dual result involving $(R, S)$-injectivity instead of $(R, S)$-projectivity, one can easily establish the following results, which are quite analogous to standard facts concerning $\operatorname{Ext}_{R}^{n}$. Every pair of $R$-homomorphisms $A \rightarrow A^{\prime}, B \rightarrow B^{\prime}$ induces a unique homomorphism $\operatorname{Ext}_{(R, S)}^{n}\left(A^{\prime}, B\right)$ $\rightarrow \operatorname{Ext}_{(R, S)}^{n}\left(A, B^{\prime}\right)$, and these induced homomorphisms have all the requisite properties to make $\operatorname{Ext}_{(R, S)}^{n}$ a functor, contravariant in the first argument, 
and covariant in the second. Moreover, the actual choice of the resolutions of $A$ and $B$ that are used in computing $\operatorname{Ext}_{(R, S)}^{n}(A, B)$ has, to within natural isomorphisms, no influence on the result. In particular, it follows that, for $n>0, \operatorname{Ext}_{(R, S)}^{n}(A, B)=(0)$, whenever either $A$ is $(R, S)$-projective or $B$ is $(R, S)$-injective. On the other hand, it can be seen directly from the definition that, for arbitrary $A$ and $B, \operatorname{Ext}_{(R, S)}^{0}(A, B)=\operatorname{Hom}_{R}(A, B)$.

Now let $X^{\prime}$ be the subcomplex of $X$ that we have used above in reducing the computation of $\operatorname{Tor}_{n}^{(R, S)}(A, B)$. Since the components of $Y$ are $(R, S)$ injective, the $(R, S)$-exact sequence $(0) \rightarrow X^{\prime} \rightarrow X \rightarrow A \rightarrow(0)$ induces an exact sequence of complexes $(0) \rightarrow \operatorname{Hom}_{R}(A, Y) \rightarrow \operatorname{Hom}_{R}^{\prime}(X, Y) \rightarrow \operatorname{Hom}_{R}^{\prime}\left(X^{\prime}, Y\right) \rightarrow(0)$, where the prime in Hom' indicates that we take the weak direct sum of the homomorphism groups for the homogeneous components rather than the full group of all homomorphisms. Define a decreasing sequence of subcomplexes $Z_{k}$ of $\operatorname{Hom}_{R}^{\prime}\left(X^{\prime}, Y\right)$ by taking $Z_{k}^{n}=\sum_{j \geqq k} \operatorname{Hom}_{R}\left(X_{n-\jmath}^{\prime}, Y^{j}\right)$. Now we can proceed in almost the same way as in our discussion of $X^{\prime} \otimes_{R} Y$ above and conclude that the cohomology groups of the complex $\operatorname{Hom}_{R}^{\prime}\left(X^{\prime}, Y\right)$ are (0). Hence the map $\operatorname{Hom}_{R}(A, Y) \rightarrow \operatorname{Hom}_{R}^{\prime}(X, Y)$ induces an isomorphism of $H^{n}\left(\operatorname{Hom}_{R}(A, Y)\right)$ onto $H^{n}\left(\operatorname{Hom}_{R}^{\prime}(X, Y)\right)=\operatorname{Ext}_{(R, S)}^{n}(A, B)$.

Similarly, let $Y^{\prime}$ denote the factor complex of $Y$ modulo the image of $B$ in $Y^{0}$. We then have the $(R, S)$-exact sequence $(0) \rightarrow B \rightarrow Y \rightarrow Y^{\prime} \rightarrow(0)$. Since each component of $X$ is $(R, S)$-projective, the induced sequence of complexes $(0) \rightarrow \operatorname{Hom}_{R}^{\prime}(X, B) \rightarrow \operatorname{Hom}_{R}^{\prime}(X, Y) \rightarrow \operatorname{Hom}_{R}^{\prime}\left(X, Y^{\prime}\right) \rightarrow(0)$ is exact. Define a decreasing sequence of subcomplexes $T_{k}$ of $\operatorname{Hom}_{R}^{\prime}\left(X, Y^{\prime}\right)$ by taking $T_{k}^{n}$ $=\sum_{i \geqq k} \operatorname{Hom}_{R}\left(X_{i}, Y^{\prime n-i}\right)$. Proceeding as before, one now shows that the cohomology groups of the complex $\operatorname{Hom}_{R}^{\prime}\left(X, Y^{\prime}\right)$ are (0). Hence the map $\operatorname{Hom}_{R}^{\prime}(X, B) \rightarrow \operatorname{Hom}_{R}^{\prime}(X, Y)$ induces an isomorphism of $H^{n}\left(\operatorname{Hom}_{R}^{\prime}(X, B)\right)$ onto $H^{n}\left(\operatorname{Hom}_{R}^{\prime}(X, Y)\right)$. We may therefore identify $\operatorname{Ext}_{(R, S)}^{n}(A, B)$ with $H^{n}\left(\operatorname{Hom}_{R}^{\prime}(X, B)\right)$, as well as with $H^{n}\left(\operatorname{Hom}_{R}(A, Y)\right)$.

Now let $(0) \rightarrow U \rightarrow V \rightarrow W \rightarrow(0)$ be an $(R, S)$-exact sequence. This leads to exact sequences of complexes

$$
(0) \rightarrow \operatorname{Hom}_{R}^{\prime}(X, U) \rightarrow \operatorname{Hom}_{R}^{\prime}(X, V) \rightarrow \operatorname{Hom}_{R}^{\prime}(X, W) \rightarrow(0),
$$

and

$$
(0) \rightarrow \operatorname{Hom}_{R}(W, Y) \rightarrow \operatorname{Hom}_{R}(V, Y) \rightarrow \operatorname{Hom}_{R}(U, Y) \rightarrow(0) .
$$

Using our reduced expressions for $\operatorname{Ext}_{(R, S)}^{n}$, we see that the first of these sequences yields an exact sequence

$\rightarrow \operatorname{Ext}_{(R, S)}^{n}(A, U) \rightarrow \operatorname{Ext}_{(R, S)}^{n}(A, V) \rightarrow \operatorname{Ext}_{(R, S)}^{n}(A, W) \rightarrow \operatorname{Ext}_{(R, S)}^{n+1}(A, U) \rightarrow$.

Similarly, the second sequence yields an exact sequence

$$
\rightarrow \operatorname{Ext}_{(R, S)}^{n}(W, B) \rightarrow \operatorname{Ext}_{(R, S)}^{n}(V, B) \rightarrow \operatorname{Ext}_{(R, S)}^{n}(U, B) \rightarrow \operatorname{Ext}_{(R, S)}^{n+1}(W, B) \rightarrow .
$$


In particular, consider the $(R, S)$-exact sequence $(0) \rightarrow K_{A} \rightarrow R \otimes{ }_{S} A \rightarrow A$ $\rightarrow(0)$. This gives rise to the exact sequence $\operatorname{Hom}_{R}\left(R \otimes_{S} A, B\right) \rightarrow \operatorname{Hom}_{R}\left(K_{A}, B\right)$ $\rightarrow \operatorname{Ext}_{(R, S)}^{1}(A, B) \rightarrow(0)$. For every $h \in \operatorname{Hom}_{R}\left(K_{A}, B\right)$, let $I_{h}$ denote the $R$ submodule of the direct sum $B+R \otimes{ }_{S} A$ that consists of all elements of the form $h(k)+k$, with $k \in K_{A}$. Put $E_{h}=\left(B+R \otimes_{S} A\right) / I_{h}$. The natural map of $B$ into $E_{h}$ is a monomorphism, so that we may identify $B$ with its image in $E_{h}$. The projection of $B+R \otimes_{S} A$ onto $R \otimes{ }_{S} A$, followed by the natural map $R \otimes{ }_{S} A \rightarrow A$, induces an $R$-epimorphism $E_{h} \rightarrow A$ whose kernel coincides with $B$. The map that sends each element $a \in A$ onto the coset $\bmod I_{h}$ of $1 \otimes a \in R \otimes s A$ is an $S$-monomorphism $A \rightarrow E_{h}$, and its composite with our epimorphism $E_{h} \rightarrow A$ is the identity map on $A$. Thus we have attached to $h$ an $S$-trivial extension of the $R$-module $B$ by the $R$-module $A$, i.e., an $(R, S)$-exact sequence (0) $\rightarrow B \rightarrow E_{h} \rightarrow A \rightarrow(0)$. It is not difficult to check that this yields a homomorphism of $\operatorname{Hom}_{R}\left(K_{A}, B\right)$ onto the group (with the Baer composition) of the equivalence classes of the $S$-trivial extensions of $B$ by $A$. Moreover, the kernel of this homomorphism turns out to be exactly the natural image of $\operatorname{Hom}_{R}\left(R \otimes_{S} A, B\right)$ in $\operatorname{Hom}_{R}\left(K_{A}, B\right)$. Hence we conclude that $\operatorname{Ext}_{(R, S)}^{1}(A, B)$ is isomorphic with the group of the equivalence classes of the $S$-trivial extensions of $B$ by $A$.

Finally, let us observe that, in the case where the subring $S$ is semisimple, in the sense that every unitary $S$-module is semisimple (or, equivalently, that $S$ is semisimple as an $S$-module), every $R$-exact sequence is automatically $(R, S)$-exact, whence we have, for all $n$, and all modules $A, B, \operatorname{Tor}_{n}^{(R, S)}(A, B)$ $=\operatorname{Tor}_{n}^{R}(A, B)$, and $\operatorname{Ext}_{(R, S)}^{n}(A, B)=\operatorname{Ext}_{R}^{n}(A, B)$.

3. Relative cohomology of rings and algebras. Let $K$ be a commutative ring, and let $P$ be an algebra over $K$. We suppose that $P$ has an identity element, 1 , and that $Q$ is a $K$-subalgebra of $P$ such that $1 \in Q$. We denote by $P^{\prime}$ the $K$-algebra anti-isomorphic with $P$, and by $p \rightarrow p^{\prime}$ the algebra anti-isomorphism of $P$ onto $P^{\prime}$. Put $R=P \otimes_{K} P^{\prime}$, with its natural structure of an algebra over $K$. Let $S$ denote the natural image of $Q \otimes_{K} P^{\prime}$ in $R$. We regard $P$ as an $R$-module such that, for $p_{1}, p_{2}, p$ in $P,\left(p_{1} \otimes p_{2}^{\prime}\right) \cdot p=p_{1} p p_{2}$.

For any (unitary) $R$-module $M$, we define the relative cohomology group $H^{n}(P, Q, M)$ for $(P, Q)$ in $M$ as $\operatorname{Ext}_{(R, S)}^{n}(P, M)$. In order to interpret and handle some of these groups, we shall give an explicit $(R, S)$-projective resolution of $P$.

For $n \geqq 0$, let $X_{n}$ stand for the tensor product $P \otimes_{Q} \cdots \otimes_{Q} P$, with $n+2$ factors $P$. The $R$-module structure of $X_{n}$ is such that, for $a, b, p_{i}$ in $P$, $\left(a \otimes b^{\prime}\right) \cdot\left(p_{0} \otimes \cdots \otimes p_{n+1}\right)=a p_{0} \otimes p_{1} \otimes \cdots \otimes p_{n} \otimes p_{n+1} b$. It is easily seen that there is an $R$-isomorphism of $R \otimes_{S} X_{n-1}$ onto $X_{n}$ (where, momentarily, $X_{-1}$ is to stand for $P$ ) sending $\left(a \otimes b^{\prime}\right) \otimes\left(p_{0} \otimes \cdots \otimes p_{n}\right)$ into $a \otimes p_{0} \otimes \cdots$ $\otimes p_{n-1} \otimes p_{n} b$, and whose inverse sends $p_{0} \otimes \cdots \otimes p_{n+1}$ into $\left(p_{0} \otimes p_{n+1}^{\prime}\right)$ $\otimes\left(p_{1} \otimes \cdots \otimes p_{n} \otimes 1\right)$. Hence each $X_{n}$ is $(R, S)$-projective. Let $u_{0}$ be the $R$-homomorphism of $X_{0}$ onto $P$ such that $u_{0}\left(a \otimes b^{\prime}\right)=a b$. For $n>0$, let $u_{n}$ be 
the $R$-homomorphism of $X_{n}$ into $X_{n-1}$ for which

$$
u_{n}\left(p_{0} \otimes \cdots \otimes p_{n+1}\right)=\sum_{i=0}^{n}(-1)^{i}\left(p_{0} \otimes \cdots \otimes p_{i} p_{i+1} \otimes \cdots \otimes p_{n+1}\right) .
$$

It is easily checked that $u_{n-1} \circ u_{n}=0$. In order to see that we have indeed an $(R, S)$-projective resolution of $P$, we merely have to exhibit an $S$-homotopy, $h$. Define $h_{-1}: P \rightarrow X_{0}$ by $h_{-1}(p)=1 \otimes p$. For $n \geqq 0$, define $h_{n}: X_{n} \rightarrow X_{n+1}$ such that $h_{n}\left(p_{0} \otimes \cdots \otimes p_{n+1}\right)=1 \otimes p_{0} \otimes \cdots \otimes p_{n+1}$. The verification that this is actually an $S$-homotopy presents no difficulties.

An $R$-module $M$ may be regarded as a two-sided $P$-module, and we shall treat it as such in our notation, whenever this is convenient. The cochain complex with the groups $\operatorname{Hom}_{R}\left(X_{n}, M\right)$ may be identified with the cochain complex with groups $C^{n}(P, Q, M)$ consisting of all $n$-linear (with respect to $K$ ) functions $f$ from $P$ to $M$ such that, for $q \in Q$ and $p_{i} \in P$,

$$
\begin{aligned}
q \cdot f\left(p_{1}, \cdots, p_{n}\right) & =f\left(q p_{1}, p_{2}, \cdots, p_{n}\right), \\
f\left(p_{1}, \cdots, p_{i} q, p_{i+1}, \cdots, p_{n}\right) & =f\left(p_{1}, \cdots, p_{i}, q p_{i+1}, \cdots, p_{n}\right), \\
f\left(p_{1}, \cdots, p_{n} q\right) & =f\left(p_{1}, \cdots, p_{n}\right) \cdot q .
\end{aligned}
$$

Here, $C^{0}(P, Q, M)$ is to be the group of all elements $m \in M$ for which $q \cdot m$ $=m \cdot q$, for all $q \in Q$. The coboundary operator $\delta: C^{n}(P, Q, M) \rightarrow C^{n+1}(P, Q, M)$ is given by the usual formula

$$
\begin{aligned}
(\delta f)\left(p_{1}, \cdots, p_{n+1}\right) & =p_{1} \cdot f\left(p_{2}, \cdots, p_{n+1}\right) \\
+ & \sum_{i=1}^{n}(-1)^{i} f\left(p_{1}, \cdots, p_{i} p_{i+1}, \cdots, p_{n+1}\right) \\
+ & (-1)^{n+1} f\left(p_{1}, \cdots, p_{n}\right) \cdot p_{n+1} .
\end{aligned}
$$

These relative cohomology groups can be used to obtain generalizations of the results of the ordinary cohomology theory for algebras (which is the case where $K$ is a field and $Q$ coincides with $K$ ). One of the simplest of these is as follows.

Theorem 1. Suppose that $H^{1}(P, Q, M)=(0)$, for all $P \otimes_{K} P^{\prime}$-modules $M$. Then, if a $P \otimes_{K} P^{\prime}$-module is semisimple for its induced structure of a $Q \otimes_{K} Q^{\prime}$ module, it is semisimple also as a $P \otimes_{K} P^{\prime}$-module.

Proof. Let $V$ be a $P \otimes_{K} P^{\prime}$-module satisfying the condition of the theorem, and let $U$ be a submodule of $V$. Put $W=V / U$, and let $t$ denote the natural epimorphism $V \rightarrow W$. Since $V$ is $Q \otimes_{K} Q^{\prime}$-semisimple, there exists a $Q \otimes_{K} Q^{\prime}$ monomorphism $h: W \rightarrow V$ such that $t \circ h$ is the identity map on $W$. To every $p \in P$ let us associate a map $h_{p}$ of $W$ into $U$ by setting $h_{p}(w)=h(w) \cdot p-h(w \cdot p)$. Then $h_{p} \in \operatorname{Hom}_{Q}(W, U)$. Now $\operatorname{Hom}_{Q}(W, U)$ can be given the structure of a two sided $P$-module such that, for $p \in P$ and $g \in \operatorname{Hom}_{Q}(W, U),(p \cdot g)(w)$ $=g(w \cdot p)$ and $(g \cdot p)(w)=g(w) \cdot p$. Moreover, remembering that $Q$ contains the 
$K$-multiples of the identity element of $P$, and that our modules are unitary, it is easily verified that this actually makes $\operatorname{Hom}_{Q}(W, U)$ into a $P \otimes_{K} P^{\prime}$. module. Now one verifies directly that the map $p \rightarrow h_{p}$ is an element $h^{\prime}$ of $C^{1}\left(P, Q, \operatorname{Hom}_{Q}(W, U)\right)$, and that $\delta h^{\prime}=0$. By the assumption of our theorem, there exists an element $g$ in $C^{0}\left(P, Q, \operatorname{Hom}_{Q}(W, U)\right)$ such that $\delta g=h^{\prime}$. This means that $g$ is an element of $\operatorname{Hom}_{Q}(W, U)$ such that $q \cdot g=g \cdot q$, for all $q \in Q$, and $h(w) \cdot p-h(w \cdot p)=g(w \cdot p)-g(w) \cdot p$, for all $p \in P$ and $w \in W$. Clearly, $h+g$ is therefore a $Q \otimes_{K} P^{\prime}$-homomorphism of $W$ into $V$, and $t \circ(h+g)$ is the identity map on $W$. Put $f=h+g$ and, for every $p \in P$, let $f_{p}$ be the map of $W$ into $U$ that sends each $w \in W$ into $f_{p}(w)=f(p \cdot w)-p \cdot f(w)$. Evidently, $f_{p} \in \operatorname{Hom}_{P^{\prime}}(W, U)$. We make $\operatorname{Hom}_{P^{\prime}}(W, U)$ into a two sided $P$-module such that, for $p \in P$ and $k \in \operatorname{Hom}_{P^{\prime}}(W, U),(p \cdot k)(w)=p \cdot k(w)$ and $(k \cdot p)(w)$ $=k(p \cdot w)$. It is easily seen that this actually makes $\operatorname{Hom}_{P^{\prime}}(W, U)$ into a $P \otimes_{K} P^{\prime}$-module. Now one verifies directly that the map $p \rightarrow f_{p}$ is an element $f^{\prime}$ of $C^{1}\left(P, Q, \operatorname{Hom}_{P^{\prime}}(W, U)\right)$ and that $\delta f^{\prime}=0$. Using the assumption of the theorem once more, we conclude that there is an element $k$ in $\operatorname{Hom}_{P^{\prime}}(W, U)$ such that $f(p \cdot w)-p \cdot f(w)=p \cdot k(w)-k(p \cdot w)$. Clearly, $f+k$ is a $P \otimes_{K} P^{\prime}$. homomorphism of $W$ into $V$, and $t \circ(f+k)$ is the identity map on $W$. Thus $U$ is a direct $P \otimes_{K} P^{\prime}$-module summand of $V$, and, since $U$ is an arbitrary submodule, we have proved that $V$ is semisimple.

Observe that, with $R$ and $S$ as before, the last part of our proof has actually shown that every $(R, S)$-exact sequence of $R$-modules is also $(R, R)$ exact. Conversely, this property implies that $H^{n}(P, Q, M)=(0)$, for all $M$ and all $n>0$, as is seen at once by applying it to an $(R, S)$-projective resolution of $P$.

In particular, the assumption of Theorem 1 is satisfied if $P$ is the ring of all $m$ by $m$ matrices with elements in $Q$. Indeed, let $p_{i j}$ be the usual matrix units, and let $f$ be an element of $C^{1}(P, Q, M)$ such that $\delta f=0$. Put $u=\sum_{i=1}^{m} p_{i 1} \cdot f\left(p_{1 i}\right)$. Then it can be verified directly that $u \in C^{0}(P, Q, M)$, and that $\delta u=f$, which proves our assertion. Using also the above remark, we conclude that if $P$ is a full matrix ring over $Q$ then $H^{n}(P, Q, M)=(0)$, for all $n>0$ and all $P \otimes_{K} P^{\prime}$-modules $M$.

We shall now obtain some general results connecting relative cohomological dimension with relative global ring dimension. These results are relativized versions of results of Cartan-Eilenberg (cf. Proposition 4 and its corollaries, in [4]) and actually represent the situation in a smoother form, in that no special assumptions need be made.

Let $P, Q$ be as above, and let $T$ be another $K$-algebra with identity element. Let $A$ and $B$ be $T \otimes_{K} P^{\prime}$-modules, written as left $T$ - and right $P$-modules. We define the structure of a two sided $P$-module on $\operatorname{Hom}_{T}(A, B)$ such that, for $a \in A, p \in P, h \in \operatorname{Hom}_{T}(A, B)$, we have $(p \cdot h)(a)=h(a \cdot p)$ and $(h \cdot p)(a)=h(a) \cdot p$. It is easily checked that this actually makes $\operatorname{Hom}_{T}(A, B)$ into a $P \otimes_{K} P^{\prime}$-module. 
THEOREM 2. $H^{n}\left(P, Q, \operatorname{Hom}_{T}(A, B)\right)$ is naturally isomorphic with $\operatorname{Ext}_{(E, F)}^{n}(A, B)$, where $E=T \otimes_{K} P^{\prime}$ and $F$ is the natural image of $T \otimes_{K} Q^{\prime}$ in $E$.

For the proof we require the following lemma:

Lemma 3. If $A$ is $(E, F)$-projective then $\operatorname{Hom}_{T}(A, B)$ is $(R, S)$-injective.

Proof. Let $X$ and $Y$ be $R$-modules (treated as two sided $P$-modules), and let $v$ be an $S$-homomorphism of $X$ into $Y$. Then $v$ induces the homomorphism $v^{\prime}: \operatorname{Hom}_{P^{\prime}}(Y, B) \rightarrow \operatorname{Hom}_{P^{\prime}}(X, B)$, where $v^{\prime}(h)=h \circ v$. Now $\operatorname{Hom}_{P^{\prime}}(X, B)$, and similarly the other such groups, are $E$-modules, with the following left $T$ and right $P$-module structures: $(t \cdot h)(x)=t \cdot h(x)$ and $(h \cdot p)(x)=h(p \cdot x)$. Clearly, $v^{\prime}$ is then a $T$-homomorphism. Moreover, with $q \in Q$, we have $((h \circ v) \cdot q)(x)=(h \circ v)(q \cdot x)=h(q \cdot v(x))=((h \cdot q) \circ v)(x)$, which shows that $v^{\prime}$ is also a right $Q$-homomorphism. Hence $v^{\prime}$ is an $F$-homomorphism. If $v$ is not only an $S$-homomorphism, but even an $R$-homomorphism, it follows in exactly the same way that $v^{\prime}$ is an $E$-homomorphism.

Now let $(0) \rightarrow U \rightarrow V \rightarrow W \rightarrow(0)$ be an $(R, S)$-exact sequence. Then it follows from what we have just seen, applied to the homomorphisms of the given sequence, and also to those of an $S$-homotopy, that the induced sequence

$$
(0) \rightarrow \operatorname{Hom}_{P^{\prime}}(W, B) \rightarrow \operatorname{Hom}_{P^{\prime}}(V, B) \rightarrow \operatorname{Hom}_{P^{\prime}}(U, B) \rightarrow(0) \text { is }(E, F) \text {-exact. }
$$

Next let us observe that there is a natural isomorphism between $\operatorname{Hom}_{R}\left(U, \operatorname{Hom}_{T}(A, B)\right.$ ) and $\operatorname{Hom}_{E}\left(A, \operatorname{Hom}_{P}{ }^{\prime}(U, B)\right.$ ) (and similarly with $V$ or $W$ in the place of $U)$. In fact, if $h \in \operatorname{Hom}_{R}\left(U, \operatorname{Hom}_{T}(A, B)\right)$ the corresponding element of $\operatorname{Hom}_{E}\left(A, \operatorname{Hom}_{P}^{\prime}(U, B)\right)$ is $h^{\prime}$, where $h^{\prime}(a)(u)=h(u)(a)$. It can be checked quite directly that the correspondence between $h$ and $h^{\prime}$ is indeed a natural isomorphism between the two composite Hom functors.

Since $A$ is $(E, F)$-projective, the epimorphism $\operatorname{Hom}_{P^{\prime}}(V, B) \rightarrow \operatorname{Hom}_{P^{\prime}}(U, B)$ of the $(E, F)$-exact sequence derived above induces an epimorphism $\operatorname{Hom}_{E}\left(A, \operatorname{Hom}_{P}^{\prime}(V, B)\right) \rightarrow \operatorname{Hom}_{E}\left(A, \operatorname{Hom}_{P}(U, B)\right)$. Using the natural isomorphism just observed, we conclude that the $\operatorname{map}_{H_{0}}\left(V, \operatorname{Hom}_{T}(A, B)\right.$ ) $\rightarrow \operatorname{Hom}_{R}\left(U, \operatorname{Hom}_{T}(A, B)\right)$ that is induced by the map $U \rightarrow V$ of the given $(R, S)$-exact sequence is an epimorphism. Thus $\operatorname{Hom}_{T}(A, B)$ is $(R, S)$ injective, and Lemma 3 is proved.

Now let $\cdots \rightarrow X_{1} \rightarrow X_{0} \rightarrow A \rightarrow(0)$ be an $(E, F)$-projective resolution of $A$. This induces the sequence

$$
(0) \rightarrow \operatorname{Hom}_{T}(A, B) \rightarrow \operatorname{Hom}_{T}\left(X_{0}, B\right) \rightarrow \operatorname{Hom}_{T}\left(X_{1}, B\right) \rightarrow \cdots \text {. }
$$

Apart from the fact that the roles of $(R, S)$ and $(E, F)$ are interchanged, this situation is the same as that in the first part of our proof of Lemma 3 . We conclude that the sequence connecting the $\operatorname{Hom}_{T}$ is $(R, S)$-exact, and, by Lemma 3, is therefore an $(R, S)$-injective resolution of $\operatorname{Hom}_{T}(A, B)$. It follows that $H^{n}\left(P, Q, \operatorname{Hom}_{T}(A, B)\right)$ is naturally isomorphic with the 
$n$th cohomology group of the complex $\operatorname{Hom}_{R}^{\prime}\left(P, \operatorname{Hom}_{T}^{\prime}(X, B)\right)$. On the other hand, the map $h \rightarrow h(1)$ is easily seen to be a natural isomorphism of $\operatorname{Hom}_{R}\left(P, \operatorname{Hom}_{T}\left(X_{i}, B\right)\right)$ onto $\operatorname{Hom}_{E}\left(X_{i}, B\right)$; the inverse map is $k \rightarrow k^{\prime}$, where $k^{\prime}(p)(x)=k(x \cdot p)=k(x) \cdot p$. Hence the complex $\operatorname{Hom}_{R}\left(P, \operatorname{Hom}_{T}^{\prime}(X, B)\right)$ is naturally isomorphic with the complex $\operatorname{Hom}_{E}^{\prime}(X, B)$. This means that $H^{n}\left(P, Q, \operatorname{Hom}_{T}(A, B)\right)$ may be identified with the $n$th cohomology group of the complex $\operatorname{Hom}_{E}^{\prime}(X, B)$, i.e., with $\operatorname{Ext}_{(E, F)}^{n}(A, B)$. This completes the proof of Theorem 2.

Let $c(P, Q)$ denote the largest non-negative integer $n$ (or $\infty$, if there is no largest such $n$ ) for which there exists a $P \otimes_{K} P^{\prime}$-module $M$ with $H^{n}(P, Q, M)$ $\neq(0)$. We call $c(P, Q)$ the relative cohomology dimension of the pair $(P, Q)$. On the other hand, let $d(P, Q)$ denote the relative global dimension of the pair $(P, Q)$, i.e., the largest non-negative integer $n$ (or $\infty$, if there is no largest such $n)$ for which there are $P$-modules $U$ and $V$ with $\operatorname{Ext}_{(P, Q)}^{n}(U, V) \neq(0)$. We shall also have to deal with $d(R, S)$, where $R=P \otimes_{K} P^{\prime}$ and $S$ is the natural image in $R$ of $Q \otimes_{K} P^{\prime}$. Here (and similarly later), where there is an evident natural map $Q \otimes_{K} P^{\prime} \rightarrow P \otimes_{K} P^{\prime}$ underlying our definition, we shall write $d\left(P \otimes_{K} P^{\prime}, Q \otimes_{K} P^{\prime}\right)$ instead of $d(R, S)$, whenever we wish to make a result quite explicit. Note that it follows immediately from the definitions that $c(P, Q) \leqq d\left(P \otimes_{K} P^{\prime}, Q \otimes_{K} P^{\prime}\right)$. On the other hand, consider Theorem 2 with $T=P$. We have then $E=P \otimes_{K} P^{\prime}$, while $F$ is the natural image in $P \otimes_{K} P^{\prime}$ of $P \otimes_{K} Q^{\prime}$. Hence Theorem 2 shows at once that $c(P, Q) \geqq d\left(P \otimes_{K} P^{\prime}, P \otimes_{K} Q^{\prime}\right)$. Thus we have $d\left(P \otimes_{K} P^{\prime}, Q \otimes_{K} P^{\prime}\right) \geqq c(P, Q) \geqq d\left(P \otimes_{K} P^{\prime}, P \otimes_{K} Q^{\prime}\right)$. Now interchange $P$ and $P^{\prime}$, and accordingly also $Q$ and $Q^{\prime}$. This gives $d\left(P^{\prime} \otimes_{K} P, Q^{\prime} \otimes_{K} P\right) \geqq c\left(P^{\prime}, Q^{\prime}\right) \geqq d\left(P^{\prime} \otimes_{K} P, P^{\prime} \otimes_{K} Q\right)$. Since switching the order of the factors in a tensor product of algebras is a natural isomorphism, we have $d\left(P^{\prime} \otimes_{K} P, Q^{\prime} \otimes_{K} P\right)=d\left(P \otimes_{K} P^{\prime}, P \otimes_{K} Q^{\prime}\right)$ and $d\left(P^{\prime} \otimes_{K} P, P^{\prime} \otimes_{K} Q\right)$ $=d\left(P \otimes_{K} P^{\prime}, Q \otimes_{K} P^{\prime}\right)$. Hence our two chains of inequalities give equalities.

Corollary 1. $d\left(P \otimes_{K} P^{\prime}, Q \otimes_{K} P^{\prime}\right)=c(P, Q)=d\left(P \otimes_{K} P^{\prime}, P \otimes_{K} Q^{\prime}\right)$ $=c\left(P^{\prime}, Q^{\prime}\right)$.

Now consider Theorem 2 with $T=K$. Then $E=P^{\prime}$ and $F=Q^{\prime}$, whence we see that $c(P, Q) \geqq d\left(P^{\prime}, Q^{\prime}\right)$. Similarly, $c\left(P^{\prime}, Q^{\prime}\right) \geqq d(P, Q)$.

Corollary 2. $c(P, Q) \geqq \max \left(d(P, Q), d\left(P^{\prime}, Q^{\prime}\right)\right)$.

We are now in a position to obtain the following generalization of a result due to Cartan-Eilenberg [2, Chapter IX, Theorem 7.11].

TheOREM 3. Let $Q$ be a commutative ring with identity element, and let $P=Q\left[x_{1}, \cdots, x_{n}\right]$ be the ring of polynomials in $n$ variables with coefficients in $Q$. Then $d(P, Q)=n=c(P, Q)$.

Proof. We regard $Q$ as a $P$-module such that, for $q \in Q$ and $p\left(x_{1}, \cdots, x_{n}\right)$ $\in P$, we have $p\left(x_{1}, \cdots, x_{n}\right) \cdot q=p(0, \cdots, 0) q$. We shall use a complex that was originally introduced by J. L. Koszul [6]: let $V$ denote the free $Q$-module 
of rank $n$, with the free basis $y_{1}, \cdots, y_{n}$. Let $V_{k}$ denote the homogeneous component of degree $k$ of the exterior $Q$-algebra constructed over $V$. Thus, in particular, $V_{0}=Q, V_{1}=V$, and $V_{k}=(0)$, for all $k>n$. The homogeneous component of degree $k$ of Koszul's complex is defined as $T_{k}=P \otimes_{Q} V_{k}$. Let $t_{0}$ denote the map $p\left(x_{1}, \cdots, x_{n}\right) \rightarrow p(0, \cdots, 0)$ of $P$ onto $Q$. Clearly, $t_{0}$ is a $P$-epimorphism $P=T_{0} \rightarrow Q$. Let $v \rightarrow v^{\prime}$ denote the $Q$-monomorphism $V \rightarrow P$ that sends each $y_{i}$ onto $x_{i}$. For $k>0$, let $t_{k}$ be the $P$-homomorphism of $T_{k}$ into $T_{k-1}$ that sends $p \otimes v_{1} \cdots v_{k}$, where $p \in P$ and $v_{i} \in V$, onto

$$
\sum_{i=1}^{n}(-1)^{i-1} p v_{i}^{\prime} \otimes v_{1} \cdots v_{i-1} v_{i+1} \cdots v_{k} .
$$

It is easily verified that $t_{k-1} \circ t_{k}=0$. Since each $T_{k}$ is $(P, Q)$-projective (actually, $P$-free), there remains only to exhibit an $S$-homotopy in order to conclude that $(T, t)$ is a $(P, Q)$-projective resolution of $Q$. We define $h_{-1}: Q \rightarrow T_{0}$ by putting $h_{-1}(q)=1 \otimes q$. Now observe that each $T_{k}$ is a free $Q$-module; for $k>0$, the elements $1 \otimes y_{j_{1}} \cdots y_{j_{k}}$ and $x_{i_{1}} \cdots x_{i_{q}} \otimes y_{j_{1}} \cdots y_{j_{k}}$, where $j_{1}<$ $j_{2}<\cdots<j_{k}$ and $i_{1} \leqq i_{2} \leqq \cdots \leqq i_{q}$, constitute a free $Q$-basis for $T_{k}$; and the elements $1 \otimes 1$ and $x_{i_{1}} \cdots x_{i_{q}} \otimes 1$ constitute a free $Q$-basis for $T_{0}$. Hence we can define an $S$-homomorphism $h_{k}: T_{k} \rightarrow T_{k+1}$ such that $h_{0}(1 \otimes 1)=0$, $h_{0}\left(x_{i_{1}} \cdots x_{i_{q}} \otimes 1\right)=x_{i_{1}} \cdots x_{i_{q-1}} \otimes y_{i_{q}}$, and, for $k>0$,

$$
h_{k}\left(1 \otimes y_{i_{1}} \cdots y_{j_{k}}\right)=0 \text {, }
$$

while

$$
h_{k}\left(x_{i_{1}} \cdots x_{i_{q}} \otimes y_{j_{1}} \cdots y_{j_{k}}\right)=(-1)^{k} x_{i_{1}} \cdots x_{i_{q-1}} \otimes y_{j_{1}} \cdots y_{j_{k}} y_{i_{q}},
$$

if $i_{q}>j_{k}$, and $=0$ otherwise. It is not difficult to verify directly that $h$ is indeed a $Q$-homotopy of $(T, t)$.

Since $Q$ is annihilated by every polynomial with constant term 0 , it follows immediately from the definition of $t_{k}$ that the induced map $\operatorname{Hom}_{P}\left(T_{k-1}, Q\right) \rightarrow \operatorname{Hom}_{P}\left(T_{k}, Q\right)$ is the 0 -map, for all $k>0$. Hence, for all $k \geqq 0$, we may identify $\operatorname{Ext}_{(P, Q)}^{k}(Q, Q)$ with $\operatorname{Hom}_{P}\left(T_{k}, Q\right)$. In particular, we see that $\operatorname{Ext}\left({ }_{(P, Q)}^{n} Q, Q\right)$ is isomorphic with $Q$. Hence we have $d(P, Q) \geqq n$.

Now let $R=P\left[z_{1}, \cdots, z_{n}\right]$ be the polynomial ring in $n$ new variables with coefficients in $P$. There is an isomorphism $\chi$ of $R$ onto $P \otimes_{Q} P$ such that $\chi(p)=1 \otimes p$, for $p \in P$, and $\chi\left(z_{i}\right)=x_{i} \otimes 1-1 \otimes x_{i}$. Then $\chi(P)=1 \otimes P$, and the natural operations of $P \otimes_{Q} P\left(=P \otimes_{Q} P^{\prime}\right)$ on $P$ make $P$ into an $R$-module (via $\chi$ ) such that $r\left(z_{1}, \cdots, z_{n}\right) \cdot p=r(0, \cdots, 0) p$, for all $p \in P$ and all $r\left(z_{1}, \cdots, z_{n}\right) \in R$. Now consider the construction of the beginning of this proof, with $R$ in the place of $P$, and $P$ in the place of $Q$. It gives an $(R, P)$ projective resolution of $P$ whose components reduce to $(0)$, for degree $>n$. Through the isomorphism $\chi$, this becomes a $\left(P \otimes_{Q} P^{\prime}, Q \otimes_{Q} P^{\prime}\right)$-projective resolution of $P$. Hence we conclude that $c(P, Q) \leqq n$. Using our first result and Corollary 2 , we obtain $n \leqq d(P, Q) \leqq c(P, Q) \leqq n$, and Theorem 3 is proved. 
4. Relative homology and cohomology of groups. Let $G$ be a group, and let $K$ be a subgroup of $G$. Let $R$ be the group ring $Z(G)$ of $G$ over the ring $Z$ of the integers, and let $S=Z(K)$. If $r \rightarrow r^{\prime}$ is the coefficient sum homomorphism of $Z(G)$ onto $Z$ we regard $Z$ as an $R$-module such that $r \cdot z=r^{\prime} z$. For a unitary $R$-module $M$, we define the relative cohomology groups for $(G, K)$ in $M$ by $H^{n}(G, K, M)=\operatorname{Ext}_{(R, S)}^{n}(Z, M)$. Accordingly, we shall exhibit an $(R, S)$-projective resolution of $Z$.

For all $n \geqq 0$, let $X_{n}$ denote the free abelian group generated by the set of $(n+1)$-tuples $\left(A_{0}, \cdots, A_{n}\right)$ of cosets $A_{i}=g_{i} K$, with $g_{i} \in G$. The $R$-module structure of $X_{n}$ is such that, for $g \in G, g \cdot\left(A_{0}, \cdots, A_{n}\right)=\left(g A_{0}, \cdots, g A_{n}\right)$. Agreeing momentarily that $X_{-1}=Z$, there is an $R$-isomorphism of $R \otimes_{s} X_{n-1}$ onto $X_{n}$ sending $g \otimes\left(A_{1}, \cdots, A_{n}\right)$ onto $\left(g K, g A_{1}, \cdots, g A_{n}\right)$; its inverse sends $\left(A_{0}, \cdots, A_{n}\right)$ onto $g_{0} \otimes\left(g_{0}^{-1} A_{1}, \cdots, g_{0}^{-1} A_{n}\right)$, where $g_{0}$ is any representative of $A_{0}$ in $G$, whose particular choice has no influence on the image. Hence each $X_{n}$ is $(R, S)$-projective.

We define $u_{0}$ to be the coefficient sum homomorphism of $X_{0}$ onto $Z$. For $n>0$, we define $u_{n}: X_{n} \rightarrow X_{n-1}$ such that

$$
u_{n}\left(A_{0}, \cdots, A_{n}\right)=\sum_{i=0}^{n}(-1)^{i}\left(A_{0}, \cdots, A_{i-1}, A_{i+1}, \cdots, A_{n}\right) .
$$

As is well known, $(X, u)$ is an acyclic $R$-complex, and there remains only to exhibit an $S$-homotopy, $h$. We define $h_{-1}: Z \rightarrow X_{0}$ by putting $h_{-1}(z)=z(K)$. For $n \geqq 0$, we define $h_{n}: X_{n} \rightarrow X_{n+1}$ such that $h_{n}\left(A_{0}, \cdots, A_{n}\right)=\left(K, A_{0}, \cdots\right.$, $\left.A_{n}\right)$. The verification that $h$ is then indeed an $S$-homotopy presents no difficulties.

If $M$ is an $R$-module, the cochain complex $\operatorname{Hom}_{R}^{\prime}(X, M)$ is the usual homogeneous group complex, except for the fact that cosets have taken the place of group elements.

Any $R$-module $M$ may be regarded also as a right $R$-module such that, for $g \in G$ and $m \in M$, the right transform $m \cdot g$ of $m$ by $g$ is given by $g^{-1} \cdot m$. With this being understood, we define the $n$th relative homology group $H_{n}(G, K, M)$ as $\operatorname{Tor}_{n}^{(R, S)}(M, Z)$.

In the case where $K$ is of finite index in $G$, one can proceed as in the ordinary cohomology theory for finite groups (cf. [2]) and introduce negative dimensional cohomology groups in such a way that (after $H^{0}(G, K, M)$ has been replaced by a certain factor group of itself), for every $(R, S)$-exact module sequence $(0) \rightarrow U \rightarrow V \rightarrow W \rightarrow(0)$, one has a doubly infinite exact cohomology sequence

$$
\cdots \rightarrow H^{q}(G, K, U) \rightarrow H^{q}(G, K, V) \rightarrow H^{q}(G, K, W) \rightarrow H^{q+1}(G, K, U) \rightarrow \cdots
$$

In fact, for all $n \geqq 0$, let $X_{-n-1}=\operatorname{Hom}_{Z}\left(X_{n}, Z\right)$, made into an $R$-module such that, for $h \in \operatorname{Hom}_{Z}\left(X_{n}, Z\right), g \in G$ and $x \in X_{n},(g \cdot h)(x)=h\left(g^{-1} \cdot x\right)$. Since $(X, u)$ is $(R, S)$-exact and $Z \subset S$, the induced sequence 
$(0) \rightarrow Z=\operatorname{Hom}_{Z}(Z, Z) \rightarrow \operatorname{Hom}_{Z}\left(X_{0}, Z\right)=X_{-1} \rightarrow X_{-2} \rightarrow \cdots$ is $(R, S)$-exact.

Composing this sequence with the given resolution $(X, u)$ of $Z$, we obtain a commutative and $(R, S)$-exact diagram

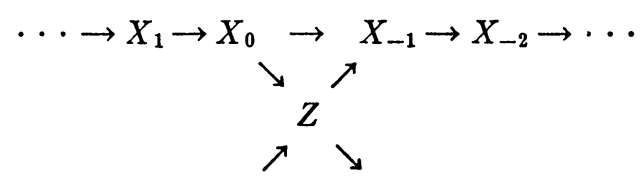

(0)

(0)

Using the natural isomorphism between $\operatorname{Hom}_{R}\left(U, \operatorname{Hom}_{Z}(X, Z)\right)$ and $\operatorname{Hom}_{R}\left(X, \operatorname{Hom}_{Z}(U, Z)\right)$, for arbitrary $R$-modules $U$ and $X$, we see easily that the $R$-modules $\operatorname{Hom}_{Z}\left(X_{n}, Z\right)(n \geqq 0)$ are $(R, S)$-injective( $\left.{ }^{2}\right)$. This has the significance that, if we make the same construction with any other $(R, S)$ projective resolution $\left(X^{\prime}, u^{\prime}\right)$ of $Z$, the resulting "complete resolution," in which $X_{-n-1}^{\prime}=\operatorname{Hom}_{Z}\left(X_{n}^{\prime}, Z\right)$, is equivalent, up to $R$-homotopies, with the one displayed above.

If $M$ is an $R$-module, we define the generalized cohomology groups $H^{q}(G, K, M)$, for $-\infty<g<\infty$, as the $q$ th cohomology groups of the complex $\operatorname{Hom}_{R}^{\prime}(X, M)$, where $X$ now stands for the weak direct sum of all the $X_{q}$, with $-\infty<q<\infty$. It follows from the remark above that these cohomology groups are still independent of the particular choice of the original $(R, S)$ projective resolution of $Z$.

Now let us make use of some of the special features of the resolution $(X, u)$ that we defined in the beginning. It is clear from our definition that (for $n \geqq 0$ ) each $X_{n}$ has a finite (because $K$ is of finite index in $G$ ) free basis over $Z$ whose elements are permuted among themselves under the action of $G$. It follows from this that $\operatorname{Hom}_{Z}\left(X_{n}, Z\right)$ is also $(R, S)$-projective. In fact, using such a basis, we can define an $R$-isomorphism of $X_{n}$ onto $\operatorname{Hom}_{Z}\left(X_{n}, Z\right)$. This is done as follows: for each basis element $x$ of $X_{n}$, let $x^{\prime}$ be the element of $\operatorname{Hom}_{Z}\left(X_{n}, Z\right)$ that takes the value 1 at $x$, but the value 0 at each of the other basis elements. Clearly, there is a $Z$-isomorphism of $X_{n}$ onto $\operatorname{Hom}_{Z}\left(X_{n}, Z\right)$ that maps each basis element $x$ onto $x^{\prime}$. Since the basis elements are permuted among themselves under the action of $G$, we have $(g \cdot x)^{\prime}=g \cdot x^{\prime}$, for every $g \in G$ and every basis element $x$. Hence our $Z$-isomorphism is actually an $R$-isomorphism.

Hence our above $(R, S)$-exact sequence connecting the $X_{q}$ for $-\infty<q<\infty$ is a complete $(R, S)$-projective resolution of $Z$, and therefore leads to the doubly infinite exact cohomology sequences announced above. It is easily seen from our definition of the $X_{n}$ that the cohomology groups $H^{q}(G, K, M)$ are precisely those defined by Adamson in [1]. Moreover, Adamson has shown that the doubly infinite exact cohomology sequence results from any

(2) The proof is similar to, but much simpler than that of Lemma 3. 
$R$-exact sequence $(0) \rightarrow U \rightarrow V \rightarrow W \rightarrow(0)$ with the property that, for every subgroup $L$ of $K$ that is an intersection of conjugates of $K$, the induced sequence $(0) \rightarrow U^{L} \rightarrow V^{L} \rightarrow W^{L} \rightarrow(0)$ of the $L$-fixed subgroups is still exact. Since this condition is evidently satisfied whenever the given sequence is $(R, S)$ exact, Adamson's result is stronger than what we have obtained above by using merely the general theory.

Next we observe that, if $K$ is of finite index in $G$, we can augment the system of homology groups for $(G, K)$ by using the same complete resolutions of $Z$. Thus, for all $-\infty<q<\infty$, we define $H_{q}(P, Q, M)$ as the $q$ th homology group of the complex whose components are the $M \otimes_{R} X_{q}$. This replaces the former $H_{0}(P, Q, M)$ by a certain subgroup of itself. If $(0) \rightarrow U \rightarrow V \rightarrow W \rightarrow(0)$ is an $(R, S)$-exact sequence, we have then a doubly infinite exact homology sequence

$$
\cdots \rightarrow H_{q}(G, K, U) \rightarrow H_{q}(G, K, V) \rightarrow H_{q}(G, K, W) \rightarrow H_{q-1}(G, K, U) \rightarrow \cdots
$$

In the ordinary theory for finite groups, one has $H^{-n}(G, M) \approx H_{n-1}(G, M)$ and $H_{-n}(G, M) \approx H^{n-1}(G, M)$, for all $n>1$ (also for $n=1$, when the modified $H^{0}$ and $H_{0}$ are used). The reason for this is that then the groups $X_{n}$ of the usual homogeneous resolution of $Z$ (take $K=(1)$, in the above) have a finite free $Z$-basis (the $(n+1)$-tuples of group elements) on which $G$ operates without fixed points, which means that the $X_{n}$ are free $R$-modules of finite rank. Now it is easily seen that a finite free $R$-basis for $X_{n}$ allows us to define, for every $R$-module $M$, isomorphisms between $\operatorname{Hom}_{R}\left(\operatorname{Hom}_{Z}\left(X_{n}, Z\right), M\right)$ and $M \otimes_{R} X_{n}$, and between $M \otimes_{R} \operatorname{Hom}_{Z}\left(X_{n}, Z\right)$ and $\operatorname{Hom}_{R}\left(X_{n}, M\right)$, these isomorphisms leading to isomorphisms between the homology and cohomology groups, as indicated above.

In the relative case, this breaks down, and we are left with separate (augmented) homology and cohomology structures. There is a very simple example showing that the positive dimensional homology groups differ from the negative dimensional cohomology groups, and also that the negative dimensional homology groups differ from the positive dimensional cohomology groups.

Let $G=K \times L$, where $K$ and $L$ are groups of order 2. Let $s$ be a generator for $K, t$ a generator for $L$. Let $Z^{\prime}$ be the $R$-module whose underlying group is the additive group $Z$ of the integers and where the operators are given by $s \cdot z=-z$, and $t \cdot z=z$, for all $z \in Z^{\prime}$. Then the $K$-fixed subgroup $Z^{\prime K}$ of our $R$-module $Z^{\prime}$ is $(0)$. Now it has been shown by Adamson (see also $\S 6$, here) that if $K$ is a normal subgroup of $G$ one has $H^{q}(G, K, M)=H^{q}\left(G / K, M^{K}\right)$, for every $R$-module $M$. Hence we have $H^{q}\left(G, K, Z^{\prime}\right)=(0)$. On the other hand, let $I(K)$ be the kernel of the coefficient sum homomorphism of $Z(K)$ onto $Z$. As we shall see in $\S 6$, we have then $H_{q}(G, K, M)=H_{q}(G / K, M / I(K) \cdot M)$. By what we have said above about the ordinary homology theory for finite groups, this last group may be identified with $H^{-q-1}(G / K, M / I(K) \cdot M)$. If 
$M=Z^{\prime}$, we have $M / I(K) \cdot M=Z / 2 Z$, with trivial $G / K$-operators. $G / K$ being cyclic, these cohomology groups are well known. In our case, they all coincide with $Z / 2 Z$.

We refer to [1] for such information on the relative cohomology groups as is significant for field theory. The relative homology groups have not been investigated, and their significance remains to be elucidated.

By way of illustration, we shall be content here with an elementary interpretation of $H^{2}(G, K, M)$ in the framework of the theory of group extensions. Let $M$ be an $R$-module. By a $(G, K)$-extension of $M$ we shall mean a pair $(E, p)$, where $E$ is a group containing both $K$ and $M$, and $p$ is a homomorphism of $E$ onto $G$ satisfying the following conditions: (1): for $e \in E$ and $m \in M, e m e^{-1}=p(e) \cdot m ;(2): p$ is the identity map on $K ;(3):$ the kernel of $p$ is $M$; (4): the identity map of $K$ into $E$ can be extended to a map $q$ of $G$ into $E$ such that $p \circ q$ is the identity map on $G$, and $q(g k)=q(g) k, q(k g)=k q(g)$, for all $g \in G$ and $k \in K$.

We shall say that two such extensions $(E, p)$ and $\left(E^{\prime}, p^{\prime}\right)$ are equivalent if there exists an isomorphism $t$ of $E$ onto $E^{\prime}$ which is the identity map on $M$ and on $K$, and such that $p^{\prime} \circ t=p$. With the usual composition, one obtains the structure of an abelian group on the set of equivalence classes of $(G, K)$ extensions of $M$. We shall show that this group is canonically isomorphic with $H^{2}(G, K, M)$.

We shall employ a shortened form of the cochain complex derived from the homogeneous $(R, S)$-projective resolution of $Z$. At the beginning of this section, we exhibited an $R$-isomorphism of $R \otimes_{S} X_{n-1}$ onto $X_{n}$. This yields an isomorphism of $\operatorname{Hom}_{R}\left(X_{n}, M\right)$ onto $\operatorname{Hom}_{R}\left(R \otimes_{S} X_{n-1}, M\right)$. Now this last group may evidently be identified with $\operatorname{Hom}_{S}\left(X_{n-1}, M\right)$, whose elements may be regarded as the functions $f$ of $n$-tuples of cosets $g K$, taking values in $M$, and such that $f\left(k A_{1}, \cdots, k A_{n}\right)=k \cdot f\left(A_{1}, \cdots, A_{n}\right)$, whenever $k \in K$. In terms of these functions, the coboundary operator turns out to be given by

$$
\begin{aligned}
(\delta f)\left(A_{1}, \cdots, A_{n+1}\right)= & g_{1} \cdot f\left(g_{1}^{-1} A_{2}, \cdots, g_{1}^{-1} A_{n+1}\right) \\
& +\sum_{i=1}^{n+1}(-1)^{i} f\left(A_{1}, \cdots, A_{i-1}, A_{i+1}, \cdots, A_{n+1}\right),
\end{aligned}
$$

where $g_{1}$ is any representative in $G$ of the coset $A_{1}$. Here, a 0 -cochain is an element $m$ of $M^{K}$, and $(\delta m)\left(A_{1}\right)=g_{1} \cdot m-m$.

Now a $(G, K)$-extension of $M$ gives rise to 2-cocycles $f$ of the type just described, as follows: $f\left(A_{1}, A_{2}\right)=q\left(g_{1}\right) q\left(g_{1}^{-1} g_{2}\right) q\left(g_{2}\right)^{-1}$, where $g_{i}$ is a representative of $A_{i}$ in $G$, whose particular choice has no influence on the expression defining $f\left(A_{1}, A_{2}\right)$. If $q^{\prime}$ is any other map satisfying the above condition (4), then $q^{\prime}(g)=r(g) q(g)$, with $r(g) \in M$. Also, for $k \in K$, we have $k r(g) q(g)=k q^{\prime}(g)$ $=q^{\prime}(k g)=r(k g) k q(g)$, whence $r(k g)=k \cdot r(g)$. On the other hand, $r(g) q(g) k$ $=q^{\prime}(g) k=q^{\prime}(g k)=r(g k) q(g) k$, whence $r(g k)=r(g)$. Hence $r$ may be regarded 
as a 1 -cochain $r^{\prime}$ for $(G, K)$ in $M$. If $f^{\prime}$ is defined from $q^{\prime}$ in the same way as $f$ was defined from $q$ we find that $f^{\prime}=f+\delta r^{\prime}$. Hence a $(G, K)$-extension of $M$ determines a unique element of $H^{2}(G, K, M)$. As in the analogous interpretation of the ordinary cohomology group $H^{2}(G, M)$, it can now be shown that this correspondence induces an isomorphism between $H^{2}(G, K, M)$ and the group of equivalence classes of the $(G, K)$-extensions of $M$.

5. Relative cohomology for Lie algebras. Let $L$ be a Lie algebra over a field $F$, and let $K$ be a subalgebra of $L$. Denote the universal enveloping algebras of $K$ and $L$ by $S$ and $R$, respectively, identifying $S$ with its canonical image in $R$. If $M$ is an $L$-module, over $F$, then the structure of a unitary $R$-module is induced on $M$ in the natural way, according to the definition of the universal enveloping algebra. We regard $F$ as a unitary $R$-module with trivial operators, i.e., such that $L \cdot F=(0)$. Now we define the relative cohomology groups for $(L, K)$ in $M$ as the groups $\operatorname{Ext}_{(R, S)}^{n}(F, M)$.

We shall give an explicit $(R, S)$-projective resolution of $F$. Let $R^{+}$denote the ideal of $R$ that is generated by the elements of $L$, and similarly define $S^{+}$. We take $X_{0}=R / R S^{+}$, noting that this $R$-module is isomorphic with $R \otimes_{S} F$, and thus $(R, S)$-projective. The canonical projection of $R$ onto $F$ induces an $R$-homomorphism $u_{0}$ of $X_{0}$ onto $F$. For $n>0$, we put $X_{n}$ $=R \otimes_{S} \cdots \otimes_{S} R \otimes_{S} R^{+} / R S^{+}$, with $n$ factors $R$. We then define the $R$ homomorphism $u_{n}: X_{n} \rightarrow X_{n-1}$ such that, for $r_{i} \in R$ and $v \in R^{+} / R S^{+}$,

$$
\begin{aligned}
u_{n}\left(r_{1} \otimes \cdots \otimes r_{n} \otimes v\right)= & \sum_{i=1}^{n-1}(-1)^{i-1} r_{1} \otimes \cdots \otimes r_{i} r_{i+1} \otimes \cdots \otimes r_{n} \otimes v \\
& +(-1)^{n-1} r_{1} \otimes \cdots \otimes r_{n-1} \otimes r_{n} \cdot v .
\end{aligned}
$$

It is easy to check that $u_{n-1} \circ u_{n}=0$, and since each $X_{n}$ is $(R, S)$-projective, there remains only to exhibit an $S$-homotopy, $h$. We define $h_{-1}: F \rightarrow R / R S^{+}$ as the restriction to $F$ of the natural $R$-homomorphism $R \rightarrow R / R S^{+}$. For $n>0$, we define $h_{n}: X_{n} \rightarrow X_{n+1}$ such that $h_{n}\left(r_{1} \otimes \cdots \otimes r_{n} \otimes v\right)=1 \otimes r_{1} \otimes \cdots$ $\otimes r_{n} \otimes v$. Finally, we define $h_{0}: X_{0} \rightarrow X_{1}$ as follows: the canonical projection $r \rightarrow r^{+}$of $R$ onto $R^{+}$, followed by the natural $R$-homomorphism $R^{+} \rightarrow R^{+} / R S^{+}$, induces an $F$-linear map $v \rightarrow v^{+}$of $R / R S^{+}$onto $R^{+} / R S^{+}$. We set $h_{0}(v)=1 \otimes v^{+}$. Then $h_{0}$ is indeed an $S$-homomorphism. For, let $r$ be a representative in $R$ of $v \in R / R S^{+}$, and let $s \in S$. Then $s \cdot v$ is the coset $\bmod R S^{+}$of $s r$. Hence $(s \cdot v)^{+}$ is the coset $\bmod R S^{+}$of $(s r)^{+}$. But $(s r)^{+}=s r^{+}+r^{0} s^{+}$, where $r^{0}$ is the component of $r$ in $F$. Hence $(s \cdot v)^{+}$is the coset $\bmod R S^{+}$of $s r^{+}$, and therefore coincides with $s \cdot v^{+}$. Hence $h_{0}$ is an $S$-homomorphism. Clearly, the other $h_{n}$ are also $S$-homomorphisms, and it is easy to verify that $h$ is indeed an $S$-homotopy.

In order to compare these new relative cohomology groups with those defined by Chevalley-Eilenberg (see [3], and [5]), we have to consider an altogether different $R$-complex. This complex arises from a relativization of the $R$-projective resolution of $F$ that is used in showing that the ordinary 
cohomology groups $H^{n}(L, M)$ coincide with the groups $\operatorname{Ext}_{R}^{n}(F, M)\left({ }^{3}\right)$. This resolution is as follows: denote by $E_{n}(L)$ the homogeneous component of degree $n$ of the exterior algebra built over the $F$-space $L$. The adjoint representation of $L$ extends to an $L$-module structure on $E_{n}(L)$ such that, for $x, x_{i}$ in $L, x \cdot\left(x_{1} \cdots x_{n}\right)=\left[x, x_{1}\right] x_{2} \cdots x_{n}+\cdots+x_{1} \cdots x_{n-1}\left[x, x_{n}\right]$. We put $X_{n}=R \otimes_{F} E_{n}(L)$. On the one hand, $X_{n}$ has then its natural $R$-module structure, derived from the ordinary multiplications in $R$. On the other hand, using the $L$-module structure of $E_{n}(L)$, we can define an $L$-module structure on $X_{n}$ whose operations commute with the operations of the natural $R$ module structure. In fact, for $x \in L$, we define the operator $t_{x}$ on $X_{n}$ such that, for $r \in R$ and $v \in E_{n}(L), t_{x}(r \otimes v)=r \otimes x \cdot v-r x \otimes v$.

The $R$-homomorphisms $u_{i}$ of the resolution of $F$ will be $R$-homomorphisms with respect to the natural $R$-module structure, in principle. However, they will also be $R$-homomorphisms with respect to the $R$-module structure that corresponds to our additional $L$-module structure. The definition of the $u_{i}$ is inductive. The map $u_{0}$ is the canonical projection of $R$ onto $F$. The map $u_{1}: X_{1} \rightarrow X_{0}$ is defined such that, for $r \in R$ and $x \in L=E_{1}(L), u_{1}(r \otimes x)=r x$. We have then evidently $u_{0} \circ u_{1}=0$, and it is easily checked that each $t_{x}$, and each operator of the natural $R$-module structure, commutes with $u_{0}$ and $u_{1}$. Now suppose that $u_{k}$ has already been defined for $k=0,1, \cdots, n-1$, where $n \geqq 2$, and that, for all $2 \leqq k \leqq n-1, r \in R, x \in L$ and $v \in E_{k-1}(L)$, we have $u_{k}(r \otimes v x)=u_{k-1}(r \otimes v) x+(-1)^{k} t_{x}(r \otimes v)$ (the meaning of the notation in the first term is that, if $u_{k-1}(r \otimes v)=\sum_{i} r_{i} \otimes v_{i}$, then $u_{k-1}(r \otimes v) x$ stands for $\left.\sum_{i} r_{i} \otimes v_{i} x\right)$. Then we attempt to define $u_{n}$ in such a way that this relation continues to hold for $k=n$. Clearly, this requirement can be met, provided only that the expression on the right depends only on $r$ and the product $v x \in E_{n}(L)$, but not separately on $v$ and $x$. By the multilinearity of the expression, and by the definition of the exterior algebra as a homomorphic image of the tensor algebra, this will follow as soon as we have shown that the expression reduces to 0 whenever $v$ is of the form $w x$, with $w \in E_{n-2}(L)$. For $n=2$, the expression then becomes $r w x \otimes x+t_{x}(r \otimes w x)=r w x \otimes x-r x \otimes w x=0$, observing that, here, $w \in F$. Now suppose that $n>2$. Then, reducing the first term, our expression can be written $\left(u_{n-2}(r \otimes w) x+(-1)^{n-1} t_{x}(r \otimes w)\right) x$ $+(-1)^{n} t_{x}(r \otimes w x)=(-1)^{n-1} t_{x}(r \otimes w) x+(-1)^{n} t_{x}(r \otimes w) x=0$. Hence $u_{n}$ can indeed be defined as desired. Evidently, $u_{n}$ is then an $R$-homomorphism with respect to the natural $R$-module structure. Also, by induction on $n$, one shows first that $u_{n}$ commutes with each $t_{x}$, and then that $u_{n-1} \circ u_{n}=0$. We omit this straightforward computation.

Now let $J$ be the ideal of $E(L)$ that is generated by the elements of $K$, and put $J_{n}=J \cap E_{n}(L)$. Let $Q_{n}$ denote the kernel of the natural homomorphism $R \otimes_{F} E_{n}(L) \rightarrow R \otimes_{S} E_{n}(L)$ (note that, through its $L$-module structure, $E_{n}(L)$

(3) This is carried out in [2, Chap. XIII]; our treatment includes the ordinary groups as a special case. 
has the structure of an $R$-module; our tensor product is taken with respect to the induced $S$-module structure of $\left.E_{n}(L)\right)$. Put $Y_{n}=R \otimes_{F} J_{n}+Q_{n}$. We shall show that $u_{n}\left(Y_{n}\right) \subset Y_{n-1}$. Evidently, $t_{z}\left(X_{n}\right) \subset Q_{n}$, for every $z \in K$. On the other hand, it is seen at once by induction on $k$ that, with $z_{i} \in K, r \in R$ and $v \in E_{n}(L)$, we have $r z_{1} \cdots z_{k} \otimes v-r \otimes z_{1} \cdots z_{k} \cdot v \in t_{K}\left(X_{n}\right)=\sum_{z \in K} t_{z}\left(X_{n}\right)$. Hence $Q_{n}=t_{K}\left(X_{n}\right)$. Since $u_{n}$ commutes with the operators $t_{z}$, it follows from this that $u_{n}\left(Q_{n}\right) \subset Q_{n-1}$. On the other hand, the inductive definition of $u_{n}$ shows at once that $u_{n}\left(R \otimes_{F} J_{n}\right) \subset R \otimes_{F} J_{n-1}+t_{K}\left(X_{n-1}\right)$, whence we may conclude that $u_{n}\left(Y_{n}\right) \subset Y_{n-1}$. Hence $u_{n}$ induces an $R$-homomorphism $q_{n}$ of $Z_{n}=X_{n} / Y_{n}$ into $Z_{n-1}=X_{n-1} / Y_{n-1}$ such that $q_{n-1} \circ q_{n}=0$. The $S$-module structure of $E_{n}(L)$ induces in the natural fashion an $S$-module structure on $E_{n}(L / K)$, and we may evidently identify $Z_{n}$ with $R \otimes_{S} E_{n}(L / K)$, concluding, in particular, that each $Z_{n}$ is $(R, S)$-projective.

Next we show that the complex of the $Z_{n}$ is acyclic. For this purpose, we define an increasing sequence of subcomplexes, as follows: let $R_{p}$ denote the set of all elements of $R$ that can be written in the form $\sum_{i} r_{i} s_{i}$, with $s_{i} \in S$, and $r_{i}$ a product of no more than $p$ elements of $L$. In particular, $R_{0}=S$, and $R_{p}=(0)$, for $p<0$. Put $Z_{n}^{p}=R_{p-n} \otimes_{S} E_{n}(L / K)\left({ }^{4}\right)$. It follows at once from the inductive definition of $u_{n}$ that $q_{n}\left(Z_{n}^{p}\right) \subset Z_{n-1}^{p}$, so that the $Z_{n}^{p}(n=0,1, \cdots)$ make up a subcomplex $Z^{p}$ of the complex of the $Z_{n}$. Now consider the factor complex $Z^{p} / Z^{p-1}$. It is easily seen that $\left(Z^{p} / Z^{p-1}\right)_{n}$ is isomorphic with $P_{p-n}(L / K) \otimes_{F} E_{n}(L / K)$, where $P_{t}(L / K)$ denotes the homogeneous component of degree $t$ of the symmetric algebra built over $L / K$. The boundary operator $\left(Z^{p} / Z^{p-1}\right)_{n} \rightarrow\left(Z^{p} / Z^{p-1}\right)_{n-1}$ that is induced by $q_{n}$ is thereby transported into an $F$-linear map of $P_{p-n}(L / K) \otimes_{F} E_{n}(L / K)$ into $P_{p-n+1}(L / K)$ $\otimes_{F} E_{n-1}(L / K)$ sending each element of the form $a \otimes v_{1} \cdots v_{n}$ onto $\sum_{i=1}^{n}(-1)^{i-1} a v_{i} \otimes v_{1} \cdots v_{i-1} v_{i+1} \cdots v_{n}$, as is seen by induction on $n$. Thus, the complex $Z^{p} / Z^{p-1}$ no longer involves the Lie algebra structure. Moreover, we know from the proof of Theorem 3 that this complex is acyclic. Hence the injections $Z^{p-1} \rightarrow Z^{p}$ induce isomorphisms of $H\left(Z^{p-1}\right)$ onto $H\left(Z^{p}\right)$. Hence each $H\left(Z^{p}\right)=(0)$, and it follows that $H(Z)=(0)$.

If $M$ is an $R$-module, the cohomology groups of the complex with the cochain groups $\operatorname{Hom}_{R}\left(Z_{n}, M\right)$ evidently coincide with the relative cohomology groups for $(L, K)$ in $M$ as defined by Chevalley-Eilenberg in [3] (see also [5]). If we knew that the complex $(Z, q)$ has an $S$-homotopy, we could therefore conclnde that these relative groups coincide with the groups $\operatorname{Ext}_{(R, S)}^{n}(F, M)$. In general, the question of the existence of an $S$-homotopy, and also that of the coincidence of the Chevalley-Eilenberg groups with the $\operatorname{Ext}_{(R, S)}^{n}(F, M)$ is not settled. However, in the case where the relative groups have a topological interpretation, as the topological cohomology groups, over the field of the

( $) R$ has an $F$-basis consisting of the ordered monomials in the elements of an ordered basis of $L$, an endsection of which is a basis of $K$. Hence each $R_{p}$ is a direct right $S$-module summand of $B$. This is used implicitly from now on. 
real numbers, of homogeneous spaces of compact analytic groups, it is possible to prove the existence of an $S$-homotopy for our complex $(Z, q)$. The algebraic essentials of this case consist in the base field being of characteristic 0 , and the subalgebra $K$ being reductive in $L$, i.e., $L$ being semisimple as a $K$-module. We then have the following result:

THEOREM 4. Let $L$ be a finite dimensional Lie algebra over a field $F$ of characteristic 0 . Let $K$ be a subalgebra of $L$, and assume that $K$ is reductive in $L$. Then the complex $(Z, q)$ defined above is an $(R, S)$-projective resolution of $F$, and hence the relative cohomology groups for $(L, K)$ in any L-module $M$ (as defined by Chevalley-Eilenberg) coincide with the groups $\operatorname{Ext}_{(R, S)}^{n}(F, M)$.

Proof. There remains only to show that the complex $(Z, q)$ has an $S$ homotopy. Since $K$ is reductive in $L, L / K$ is semisimple for its natural structure as a $K$-module. It follows (see Proposition 1 of [5]) that the $n$-fold tensor power of $L / K$ is also semisimple as a $K$-module. Since $E_{n}(L / K)$ is a homomorphic image of this $K$-module, it is semisimple. On the other hand, $R$ may be regarded as an $L$-module such that, for $z \in L$ and $r \in R, z \cdot r=z r-r z$. Let $R_{p}^{\prime}$ be the subspace of $R$ that is spanned by the products of no more than $p$ elements of $L$. Then, with respect to the $L$-module structure just defined, $R_{p}^{\prime}$ is an $L$-submodule of $R$. Moreover, as an $L$-module, $R_{p}^{\prime}$ is a homomorphic image of the sum of the homogeneous components of degrees $p$ of the tensor algebra built over $L$, with its natural structure as an $L$-module. Restricting the operator algebra to $K$, we obtain the structure of a semisimple $K$-module on this tensor algebra, because $K$ is reductive in $L$. Hence also $R_{p}^{\prime}$ is a semisimple $K$-module. Since $R$ is the sum of the submodules $R_{p}^{\prime}$, it follows that $R$ is semisimple as a $K$-module, and, in fact, is the sum of finite dimensional simple $K$-submodules. The same is therefore true for the tensor product $R \otimes_{F} E_{n}(L / K)$ (again by Proposition 1 of [5]), when this is regarded as a $K$-module with operators $a_{z}(z \in K)$ such that $a_{z}(r \otimes u)=(z r-r z)$ $\otimes u+r \otimes z \cdot u$. Now it is evident that each $a_{z}$ maps the above $Y_{n}$ into themselves. Hence the $a_{z}$ induce the structure of a $K$-module on $Z_{n}$, for which $Z_{n}$ is semisimple. We have $a_{z}(r \otimes u)=z r \otimes u+t_{z}(r \otimes u)$, and, since $t_{z}\left(X_{n}\right) \subset Y_{n}$, it follows that the $K$-module structure on $Z_{n}$ that is induced by the $a_{z}$ coincides with the $K$-module structure that corresponds to the natural $S$-module structure of $Z_{n}$. Hence $Z_{n}$ is semisimple with respect to its natural $S$-module structure. Since the complex $(Z, q)$ is acyclic, it follows that it has an $S$-homotopy, and Theorem 4 is proved.

6. Reduction of relative groups. If $K$ is a normal subgroup of a group $G$, and $M$ is a $G$-module, then the relative cohomology groups for $(G, K)$ in $M$ reduce to the ordinary cohomology groups for $G / K$ in the $K$-fixed part $M^{K}$ of $M$. An analogous reduction takes place in the case of a Lie algebra $L$ relative to an ideal $K$ of $L$. We shall absorb these known results in a more general result concerning certain $(R, S)$-projective resolutions. 
Let $R$ be a ring with 1 , and let $P$ be a central subring of $R$ such that $1 \in P$. Let $S$ be a subring of $R$ such that $P \subset S$. Suppose also that there is given a projection homomorphism $q$ of $R$ onto $P$. Let $S_{q}$ denote the kernel of the restriction of $q$ to $S$. Assume that $S_{q} R \subset R S_{q}$, i.e., that the left ideal $R S_{q}$ of $R$ is also a right ideal. We may then form the factor ring $R^{\prime}=R / R S_{q}$. We assume, finally, that $R^{\prime}$ is $P$-projective, for its natural structure as a $P$ algebra.

Using the projection $q$, we may regard $P$ as an $R$-module such that, for $p \in P$ and $r \in R, r \cdot p=q(r) p$. Now consider the standard $(R, S)$-projective resolution $(X, u)$ of $P$. One sees at once by induction on $n$ that each $X_{n}$ is annihilated by $S_{q}$, so that it may also be regarded as an $R^{\prime}$-module. We claim that, when so regarded as an $R^{\prime}$-complex, $(X, u)$ is an $R^{\prime}$-projective resolution of $P$.

In order to see this, consider $R \otimes_{S} U$, where $U$ is any $R$-module that is annihilated by $S_{q}$. The exact double $R$-module sequence $(0) \rightarrow R S_{q} \rightarrow R \rightarrow R^{\prime}$ $\rightarrow(0)$ induces the exact $R$-module sequence $R S_{q} \otimes_{S} U \rightarrow R \otimes_{S} U \rightarrow R^{\prime} \otimes_{S} U \rightarrow(0)$. Since $U$ is annihilated by $S_{q}$, the first map of this sequence is the 0-map. Hence $R \otimes_{S} U$ is isomorphic with $R^{\prime} \otimes_{S} U$. Now $R^{\prime}$ and $U$ are both annihilated by $S_{q}$, and $S / S_{q}$ is isomorphic with $P$ in such a way that corresponding elements operate in the same way on $U, R^{\prime}$. Hence we have $R^{\prime} \otimes_{S} U=R^{\prime} \otimes_{P} U$. Now suppose, furthermore, that $U$ is $P$-projective. Then $U$ is a direct $P$ module summand of a free $P$-module, $N$, say, and $R^{\prime} \otimes_{P} U$ is a direct $R^{\prime}$ module summand of $R^{\prime} \otimes_{P} N$, which is a free $R^{\prime}$-module. Hence $R^{\prime} \otimes_{P} U$ is $R^{\prime}$-projective, and therefore also $R \otimes_{S} U$, when regarded as an $R^{\prime}$-module in the natural fashion, is $R^{\prime}$-projective.

Since $P$ is annihilated by $S_{q}$ and is $P$-projective, it follows therefore that $X_{0}\left(=R \otimes_{S} P\right)$ is annihilated by $S_{q}$, and $R^{\prime}$-projective. Since $R^{\prime}$ is $P$-projective, every $R^{\prime}$-projective module is also $P$-projective. Since the complex $(X, u)$ has an $S$-homotopy, which is a fortiori a $P$-homotopy, the kernel of $u_{0}$ is a direct $P$-module summand of $X_{0}$, and hence is still $P$-projective. Hence we may now repeat the whole argument to conclude that $X_{1}$ is $R^{\prime}$-projective, and that the kernel of $u_{1}$ is $P$-projective, etc. Hence $(X, u)$ is indeed an $R^{\prime}$ projective resolution of $P$.

Now let $M$ be an $R$-module, and let $M^{\prime}$ denote the set of all elements of $M$ that are annihilated by $S_{q}$. Then $M^{\prime}$ is an $R$-submodule of $M$. Moreover, $M^{\prime}$ may be regarded as an $R^{\prime}$-module and, as such, is canonically isomorphic with $\operatorname{Hom}_{R}\left(R^{\prime}, M\right)$, where $R^{\prime}$ is regarded as a left $R$-module and a right $R^{\prime}$-module in the natural fashion. If $U$ is any $R^{\prime}$-module, regarded also as an $R$-module via the natural homomorphism of $R$ onto $R^{\prime}$, there is a natural isomorphism between $\operatorname{Hom}_{R^{\prime}}\left(U, \operatorname{Hom}_{R}\left(R^{\prime}, M\right)\right)$ and $\operatorname{Hom}_{R}(U, M)$ sending $h \in \operatorname{Hom}_{R^{\prime}}\left(U, \operatorname{Hom}_{R}\left(R^{\prime}, M\right)\right)$ onto $h_{1} \in \operatorname{Hom}_{R}(U, M)$, where $h_{1}(a)=h(a)(1)$, for every $a \in U$; the inverse sends $k$ onto $k^{\prime}$, where $k^{\prime}(a)\left(r^{\prime}\right)=k\left(r^{\prime} \cdot a\right)$. Using these isomorphisms, for $U=X_{i}(i=0,1, \cdots)$, and the isomorphism between 
$M^{\prime}$ and $\operatorname{Hom}_{R}\left(R^{\prime}, M\right)$, we find that $\operatorname{Ext}_{(R, S)}^{n}(P, M)$ is naturally isomorphic with $\operatorname{Ext}_{R^{\prime}}^{n}\left(P, M^{\prime}\right)$.

In the application to the cohomology of groups, we have $P=Z$, and $q$ is the coefficient sum homomorphism of $R=Z(G)$ onto $Z$. If $K$ is a normal subgroup of $G$, we take $S=Z(K)$ and see at once that all our above assumptions hold in this case. The result is that $H^{n}(G, K, M)$ is naturally isomorphic with $H^{n}\left(G / K, M^{K}\right)$. Moreover, if $K$ is of finite index in $G$, it is easily seen that the $R$-modules $\operatorname{Hom}_{Z}\left(X_{n}, Z\right)$, as defined in $\S 4$, may be regarded also as $R^{\prime}$ modules and, as such, are the same as those resulting when the $X_{n}$ are regarded as $R^{\prime}$-modules and the operations on $\operatorname{Hom}_{Z}\left(X_{n}, Z\right)$ are defined by referring to $G / K$. Hence the result for the cohomology groups holds also in negative dimensions.

Similarly, if $M$ is a right $R$-module, and $U$ is an $R^{\prime}$-module as before, we may identify $M \otimes_{R} U$ with $M \otimes_{R} R^{\prime} \otimes_{R^{\prime}} U$. Using this with $U=X_{i}$ $(i=0,1, \cdots)$, we find that $\operatorname{Tor}_{n}^{(R, S)}(M, P)$ is naturally isomorphic with $\operatorname{Tor}_{n}^{R^{\prime}}\left(M \otimes_{R} R^{\prime}, P\right)$. Moreover, $M \otimes_{R} R^{\prime}$ may evidently be identified with $M / M \cdot S_{q}$.

In the case of homology for groups, this shows that, if $K$ is normal in $G, H_{n}(G, K, M)$ is naturally isomorphic with $H_{n}(G / K, M / I(K) \cdot M)$, where $I(K)$ is as in $\S 4$. As above, this continues to hold for negative $n$, when $K$ is of finite index in $G$.

In the application to the cohomology of Lie algebras, we have $P=F$ (the base field), and $q$ is the projection of the universal enveloping algebra $R$ of the Lie algebra $L$ onto $F$. If $K$ is an ideal in $L$, we take $S$ to be the universal enveloping algebra of $K$, and see that all the above assumptions now hold. The result is that then $H^{n}(L, K, M)$ is naturally isomorphic with $H^{n}\left(L / K, M^{K}\right)$, where now $M^{K}$ is the set of all elements of $M$ that are annihilated by $K$. This result holds for the relative groups as proposed here, as well as for the relative groups of Chevalley-Eilenberg (in which case it is well known, of course).

\section{REFERENCES}

1. Iain T. Adamson, Cohomology theory for non-normal subgroups and non-normal fields, Proc. Glasgow Math. Assoc. vol. 2 (1954) pp. 66-76.

2. H. Cartan and S. Eilenberg, Homological algebra, Princeton, 1956.

3. C. Chevalley and S. Eilenberg, Cohomology theory of Lie groups and Lie algebras, Trans. Amer. Math. Soc. vol. 63 (1948) pp. 85-124.

4. S. Eilenberg, Algebras of cohomologically finite dimension, Comment. Math. Helv. vol. 28 (1954) pp. 310-319.

5. G. Hochschild and J.-P. Serre, Cohomology of Lie algebras, Ann. of Math. vol. 57 (1953) pp. 591-603.

6. J. L. Koszul, Sur un type d'algèbres differentielles en rapport avec la transgression, Coll. de Topologie, Bruxelles, 1950, Liège, Thone, 1951, pp. 73-81.

University of California, Berkeley, CaltF. 